\title{
Band structure tuning of Heusler compounds: Spin- and momentum-resolved electronic structure analysis of compounds with different band filling
}

\author{
S. Chernov, C. Lidig, O. Fedchenko, K. Medjanik, S. Babenkov, D. Vasilyev, M. Jourdan, G. Schönhense, and H. J. Elmers $\oplus^{*}$ \\ Institut für Physik, Johannes Gutenberg-Universität, 55099 Mainz, Germany
}

(Received 8 November 2019; revised 12 December 2020; accepted 15 January 2021; published 3 February 2021)

\begin{abstract}
Half-metallic ferromagnetic Heusler compounds represent an important class of materials for spintronic applications. We experimentally observe the dispersion of electronic bands with spin resolution in three representative Heusler compounds $\left(\mathrm{Co}_{2} \mathrm{MnGa}, \mathrm{Co}_{2} \mathrm{MnSi}\right.$, and $\left.\mathrm{Co}_{2} \mathrm{Fe}_{0.4} \mathrm{Mn}_{0.6} \mathrm{Si}\right)$. Bulk-sensitive measurements at very low photon energy are complemented by spin-integrated hard x-ray angular resolved photoemission spectroscopy. The dispersion of majority and minority electrons allows us to link exchange splitting and band filling to the number of valence electrons $N_{v}$. Photoexcitation at $h v=6.05 \mathrm{eV}$ gives access to the spin-polarization texture $\boldsymbol{P}\left(E_{B}, k_{x}, k_{y}\right)$ of the bulk bands covering a $\left(k_{x}, k_{y}\right)$ range of $60 \%$ of the Brillouin zone. We find that $\mathrm{Co}_{2} \mathrm{MnSi}_{\mathrm{and}}$ $\mathrm{Co}_{2} \mathrm{Fe}_{0.4} \mathrm{Mn}_{0.6} \mathrm{Si}$ exhibit minority band gaps of 0.5 and $0.35 \mathrm{eV}$, i.e., decreasing band gap with increasing $N_{\mathrm{V}}$ in contrast to the rigid-band expectation. For $\mathrm{Co}_{2} \mathrm{MnGa}$, the minority valence state maximum lies at approx. $0.2 \mathrm{eV}$ above $E_{\mathrm{F}}$.
\end{abstract}

DOI: 10.1103/PhysRevB.103.054407

\section{INTRODUCTION}

Heusler compounds are a class of materials comprising more than 1000 compounds [1-4]. The composition of these compounds is $A_{2} B Z$, where $A$ and $B$ represent $3 d$ transition metals and $Z$ is a main group element. This class of materials shows fascinating properties that are important for technological applications and can be widely tuned by changing the alloy stoichiometry. One of the first intriguing properties found for Heusler alloys is the occurrence of ferromagnetism in $\mathrm{Cu}_{2} \mathrm{MnAl}$, where none of the constituting elements is ferromagnetic [5]. The variety of accessible electronic and magnetic properties in combination with the existence of topological nontrivial electronic states create highly tunable multifunctional properties, including topological superconductivity and spin-structured topological surface states [6-11]. One considers Heusler compounds as ideal materials for spintronic devices, where in contrast to conventional charge-based semiconductor electronics the spin degree of freedom of electrons serves as the information carrier [12-14]. The spin degree of freedom offers many advantages such as nonvolatility and low power consumption combined with high integration densities [13-19]. Spintronic devices require injection, transport, and manipulation of the spin in thin film structures. A key parameter for this application is the spin polarization of the charge carriers. A half-metallic ferromagnet with complete spin polarization at the Fermi level represents a favorable material for spintronics [20] not only for exploiting the spin polarization of charge carriers but also for their low damping properties [21].

Here, we investigate the electronic states of the highly spin-polarized ferromagnetic Heusler compounds $\mathrm{Co}_{2} \mathrm{MnGa}$, $\mathrm{Co}_{2} \mathrm{MnSi}$, and $\mathrm{Co}_{2} \mathrm{Fe}_{0.4} \mathrm{Mn}_{0.6} \mathrm{Si}$, which are expected to have

\footnotetext{
*elmers@uni-mainz.de
}

a very similar band structure, but different degrees of band filling. By variation of the elements $Y$ and $Z$ in $\mathrm{Co}_{2} Y Z$, calculations basically predict a modification of the band filling of these Heusler compounds within an almost rigid-band structure [22-24]. In this framework, $\mathrm{Co}_{2} \mathrm{MnGa}$ is not expected to be a half-metallic ferromagnet, whereas $\mathrm{Co}_{2} \mathrm{MnSi}$ is [25-27]. Calculations also predict that the position of the Fermi energy of the latter compound can be shifted within the minority band gap by doping with $\mathrm{Fe}$ on the $\mathrm{Mn}$ site resulting in $\mathrm{Co}_{2} \mathrm{Mn}_{1-x} \mathrm{Fe}_{x} \mathrm{Si}$ [28]. In view of the broad variation of predicted properties, where for $\mathrm{Co}_{2} \mathrm{MnSi}$ the Fermi energy ranges from within the minority states to almost $1 \mathrm{eV}$ above the minority band maximum [26-33], experiments to determine the spin-resolved band dispersion seem to be of utmost importance.

Among the ferromagnetic Heusler compounds $\mathrm{Co}_{2} \mathrm{MnSi}$ has attracted the most scientific interest [23,34-44] because of its comparatively large minority-spin band gap at the Fermi level [22] and high Curie temperature of 985 K [39]. Based on magnetic tunneling junctions, interfacial spin polarizations of up to $89 \%$ at low temperature [41] were reported, which tend to be considerably reduced at room temperature [42-46]. Growing interest in the electronic structure of the magnetic Heusler compounds was initiated by the prediction and experimental evidence for topological Hopf and chain link semimetal states for $\mathrm{Co}_{2} \mathrm{MnGa}[47,48]$.

Spin-resolved photoemission spectroscopy is the method of choice for a direct determination of the dispersion of the electronic bands and their majority and minority character. With one exception, all spin-resolved experiments used excitation with photon energies in the vacuum ultraviolet (VUV) regime, yielding a high surface sensitivity due to the limited inelastic mean free path (IMFP) of the photoelectrons. For sputtered epitaxial $\mathrm{Co}_{2} \mathrm{MnSi}(100)$ thin films we measured a spin polarization of $93 \%$ at the Fermi energy (in normal emission at $h v=21.2 \mathrm{eV}$ ) [34], containing a strong contribution 
from a fully spin-polarized surface resonance close to the Fermi energy $[49,50]$. A high spin polarization has also been reported for $\mathrm{Co}_{2} \mathrm{MnGa}$ [21], whereas Ref. [51] reports a value of only 55\%. The high spin polarization for $\mathrm{Co}_{2} \mathrm{MnSi}$ has been confirmed for MBE-grown films [35] and related to low magnetic damping [21,52].

The dominance of surface states and surface resonances in the majority of existing studies was prohibitive for a quantitative determination of the spin polarization of the bulk bands near the Fermi energy. Moreover, no experiment gave information on the dispersion of the majority and minority bulk bands.

Bulk sensitivity can be achieved at the low- and highenergy end of the universal curve of the IMFP [53], i.e., at photoelectron kinetic energies of a few $\mathrm{eV}$ and towards the $\mathrm{keV}$ range. At a very low photon energy of $h v=5.9 \mathrm{eV}$ a spin-resolved photoemission experiment denied half-metallic properties for the case of ex situ prepared $\mathrm{MgO}$-capped $\mathrm{Co}_{2} \mathrm{MnSi}$ thin films [54,55]. Soft x-ray angle-resolved photoemission spectroscopy (ARPES) on sputtered $\mathrm{Co}_{2} \mathrm{MnSi}$ thin films with $\mathrm{Al}$ capping gave evidence of the surface resonance persisting at such high energies, in good agreement with photoemission calculations $[25,49,50]$. In both experiments it was unclear how much the capping layer altered the results. Photoemission results in the hard $\mathrm{x}$-ray regime $(>3 \mathrm{keV})$ revealed spectral features originating from the bulk density of states [56,57], however, without spin and angular resolution.

Recently, a spin-resolved ARPES study with high energy resolution reports on electron- and hole-doped shifts of the $\mathrm{Co}_{2} \mathrm{MnGa}$ band structure with a focus on the spin-polarized Weyl cones in this material [58]. On the theoretical side, a large number of band-structure calculations exist, comprising density-functional theory (DFT) $[26,29,32,33]$ as well as dynamical mean-field theory (DMFT) $[30,31]$ calculations. Here, we present a spin- and momentum-resolved investigation of the electronic structure of ferromagnetic Heusler compounds.

We provide a detailed comparison of our experimentally observed bulk majority and minority band dispersion using photoexcitation at $6 \mathrm{eV}$ with previously published theoretical work, indicating a general overestimation of the calculated exchange splitting.

\section{EXPERIMENTAL DETAILS}

\section{A. In situ sample preparation and experimental setup}

The epitaxial $\mathrm{Co}_{2} \mathrm{MnSi}(100), \mathrm{Co}_{2} \mathrm{Mn}_{0.6} \mathrm{Fe}_{0.4} \mathrm{Si}(100)$, and $\mathrm{Co}_{2} \mathrm{MnGa}(100)$ films were grown on $\mathrm{MgO}(100)$ substrates at room temperature using rf-magnetron sputtering in an $\mathrm{Ar}$ atmosphere at a pressure of 0.1 mbar. The film thickness in the order of a few tens of nanometers was varied by the deposition time, the stoichiometry via the distance from the sputter target. After deposition, the samples were annealed in ultrahigh vacuum at a temperature of $550^{\circ} \mathrm{C}$. The deposition parameters were optimized by systematic screening of parameter space using as a main criterion the signal-to-background ratio for direct photoemission transitions. In addition, $\mathrm{x}$-ray diffraction and energy dispersive $\mathrm{x}$-ray spectroscopy (both ex situ) were employed for analysis of the film quality; for details, see [51]. In particular, the occurrence of the (111) diffraction peak confirms the $L 2_{1}$ crystal structure for all samples investigated here. For similarly deposited films we also performed an in situ reflection high energy electron diffraction study that also confirms the $L 2_{1}$ crystal structure at the surface of the films [34,51,59]. After finishing deposition, the samples are transferred within a few minutes under UHV conditions into the sample stage of the microscope (base pressure $2 \times$ $10^{-10} \mathrm{mbar}$ ), where the spin mapping is immediately started so that the acquisition is finished within typically half an hour after deposition. The Si-containing compounds degraded visibly after a few hours, whereas for the $\mathrm{Co}_{2} \mathrm{MnGa}$ films we found a remarkably long "lifetime" of several weeks, with the spin-polarized bands still being visible.

All thin-film samples were oriented with [011] and [011] crystallographic directions along the $x$ and $y$ axis. The photon beam impinges in the $x-z$ plane at $22^{\circ}$ from the $x$ axis. The photon polarization is varied using quarter- and half-wave plates, resulting in linear $s$ and $p$ polarization or left (LCP) and right (RCP) circular polarization. Exploiting the polarization dependence of the photoemission intensity patterns, the symmetry groups of the observed bands can be probed $[60,61]$. These symmetry selection rules are exploited for the identification of overlapping bands with different spin signature.

The fast degradation of the highly reactive Heusler films demands efficient recording, especially because the $k$ dependence of the spin texture is aimed at. Time-of-flight momentum microscopy allows parallel acquisition of the photoemitted electron intensity $I\left(E_{\mathrm{B}}, k_{x}, k_{y}\right)$ as a function of transversal momentum $k_{x}$ and $k_{y}$ and binding energy $E_{\mathrm{B}}$ $[62,63]$. Figure 1(a) shows the experimental geometry. The fourth harmonic of a femtosecond Ti-sapphire laser provides femtosecond UV pulses with a photon energy of $6.05 \mathrm{eV}$ at a rate of $80 \mathrm{MHz}$. Taking into account the sample work function, binding energies of up to $1.9 \mathrm{eV}$ are accessible with a one-photon photoemission process. The microscope observes the full half space above the sample surface (polar angular range $0-90^{\circ}$ ), which defines the "photoemission horizon." In general, the strongly confined photoemission horizon at low excitation energies poses a fundamental limitation on the usefulness of low-energy photons for comprehensive valenceband mapping (despite the increased information depth). The Heusler compounds with their relatively large unit cell represent a favorable case because the corresponding Brillouin zone is quite small. The visible field of view in $k$ space at $E_{\mathrm{F}}$ is restricted to $k_{\| \max }=0.7 \AA^{-1}$, accessing $\sim 60 \%$ of the surface Brillouin zone (BZ) along the main axes $k_{x}$ and $k_{y}$ [Fig. 1(b)].

A typical spin-resolved measurement requires only $30 \mathrm{~min}$ of acquisition time. Owing to the high repetition rate of the laser, the average photoemission yield stayed well below one photoelectron per pulse, avoiding the serious space-charge problem of low repetition rate systems $[64,65]$. The detection efficiency in the straight detector branch (spin integrated data) is only limited by the maximum count rate of $\sim 5$ Mcps of the delay line detector (DLD).

\section{B. Rapid recording of the spin-polarization texture}

Spin contrast is obtained using a $\mathrm{W}(100)$ imaging spin filter [66-69] which is characterized by a very high 

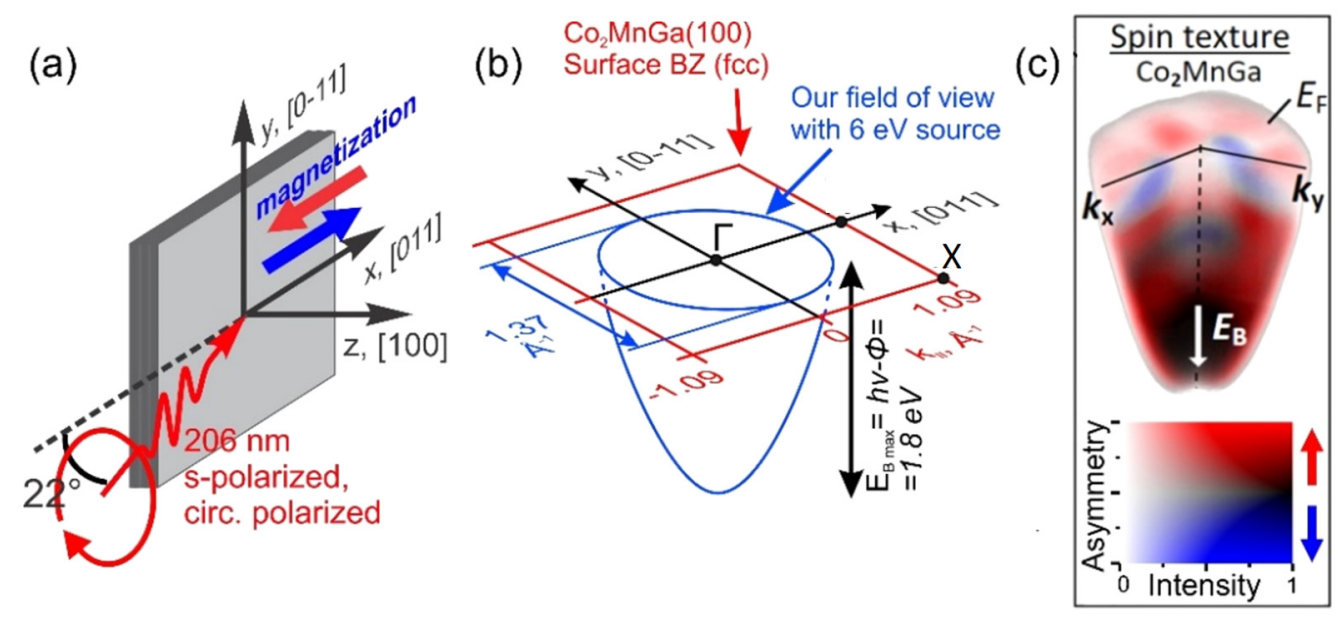

FIG. 1. Details of the photoemission experiment illustrating sample geometry and data-recording technique. (a) Sample orientation, definition of the coordinate system and impact direction of the linearly or circularly polarized photon beam. (b) Photoemission horizon defined by the kinetic-energy range and $k$ interval with $6.05-\mathrm{eV}$ photons; the paraboloid corresponds to the emission-angle range of $0-90^{\circ}$. (c) $3 \mathrm{D}$ data array showing the spin texture (component $\mathbf{P}$ ) of $\mathrm{Co}_{2} \mathrm{MnGa}$ in the complete half space above the sample surface, measured without any scanning or sample rotation. The 3D color code denotes majority (red), minority (blue), as well as unpolarized (grey) partial intensities.

reflectivity, a broad spin-asymmetry profile of $>2 \mathrm{eV}$, and the complete absence of any visible mosaicity or polycrystallinity (as observed for other spin-filter materials). The spin filter was operated at $45^{\circ}$ scattering angle and $26.5 \mathrm{eV}$ electron scattering energy; the plane of incidence was $(010)$. The corresponding single-point figure of merit is $5 \times 10^{-3}$. The spin quantization axis of the measurement points along the easy magnetization axis of the samples [011], coinciding with the $x$ axis in Fig. 1(a).

The spin-polarization texture is determined in pairs of measurements with opposite sample magnetization. Reversal of the magnetization was done in situ by approaching strong permanent magnets to the thin-film sample. The quality of the spin-filter surface is monitored by the signal-to-background ratio of the observed momentum image. Immediately before data recording the spin-filter crystal is flashed. A complete spin-texture map is measured within $\sim 0.5 \mathrm{~h}$, without visible degradation of the spin-filter surface (pressure $<10^{-9} \mathrm{mbar}$ ). The energy resolution of the experiment has been set to $20 \mathrm{meV}$. The spin-resolved $k$ resolution demonstrated here is limited by the degree of translational order of the samples.

A total of nine three-dimensional (3D) data arrays are recorded, one for each photon polarization $(s$ polarized, RCP, LCP) in the spin-integral branch and spin-filtered branch for opposite magnetization directions $M \uparrow$ and $M \downarrow$. We term these arrays $I\left(E_{\mathrm{B}}, k_{x}, k_{y}\right), I \uparrow\left(E_{\mathrm{B}}, k_{x}, k_{y}\right)$, and $I \downarrow\left(E_{\mathrm{B}}, k_{x}, k_{y}\right)$. Sum arrays for measurements with all three light polarizations partly suppress the effect of linear or circular dichroism in the photoelectron angular distribution (LDAD [62] or CDAD [61]). The (incoherent) sum of unpolarized and linearly polarized light can yield "isotropic" light, with electric vector statistically random in all spatial directions. Indeed, the residual dichroism asymmetry in the data arrays of the sum $(\mathrm{RCP}+\mathrm{LCP}+s$-pol. $)$ is very small. The symmetry selection rules can be exploited to emphasize specific bands or even resolve bands that are overlapping in the sum images. Surface states are suppressed with $s$-polarized light [21], so we show mostly measurements with $s$ polarization in the comparison with bulk calculations.

The spin-polarization texture $\mathbf{P}\left(E_{B}, k_{x}, k_{y}\right)$ is determined voxel-by-voxel from two measurements with opposite magnetization $M \uparrow$ and $M \downarrow$, with the characteristic asymmetry function of the spin filter $\mathrm{S}$ (Sherman function):

$$
\mathbf{P}\left(E_{\mathrm{B}}, k_{\mathrm{x}}, k_{\mathrm{y}}\right)=\frac{1}{\mathrm{~S}\left(E_{\mathrm{scatt}}\right)} \frac{\left[I^{\uparrow}\left(E_{\mathrm{B}}, k_{\mathrm{x}}, k_{\mathrm{y}}\right)-I^{\downarrow}\left(E_{\mathrm{B}}, k_{\mathrm{x}}, k_{\mathrm{y}}\right)\right]}{\left[I^{\uparrow}\left(E_{\mathrm{B}}, k_{\mathrm{x}}, k_{\mathrm{y}}\right)+I^{\downarrow}\left(E_{\mathrm{B}}, k_{\mathrm{x}}, k_{\mathrm{y}}\right)\right]} .
$$

In the small energy interval accessible with $6.05 \mathrm{eV}$ photons the Sherman function $\mathrm{S}=\mathrm{S}\left(E_{\text {scatt }}\right)$ is essentially independent of energy. A value of $\mathrm{S}=0.4$ has been assumed for all results shown here. All measurements have been performed at room temperature, which is well below the Curie temperatures of the compounds, lying in the range of 700-1000 K. For details on the evaluation procedure for spin-texture arrays in imaging spin filters, see [70,71].

In direct interband transitions in reciprocal space the finalstate momentum vector $\boldsymbol{k}_{\mathbf{f}}$ determines the radius of the finalstate sphere (here we consider quasi-free-electron-like final states), which intersects the periodic pattern of Brillouin zones. The radius $k_{f}$ of the energy isosphere is given by

$$
\begin{aligned}
k_{f} / \AA^{-1} & \sim 0.512 \sqrt{\left(E_{\text {final }} / e V\right)\left(m_{\text {eff }} / m\right)} \text { with } \\
E_{\text {final }} & =h v-E_{B}+V_{o}^{*},
\end{aligned}
$$

where $m_{\mathrm{eff}}$ and $m$ are the effective mass and free-electron mass, and $E_{\mathrm{B}}$ and $V_{0}{ }^{*}$ are the binding energy and inner potential, both referenced to the Fermi level. For the lattice constant of $\mathrm{Co}_{2} \mathrm{MnSi}$ [5.66(3) $\AA$ ], the reciprocal lattice vector along $k_{z}$ is $G_{100}=2.22 \AA^{-1}$. The phototransition at $h v=6.05 \mathrm{eV}$ leads to $k_{z}=2.08 \AA^{-1}=0.94 G_{100}$ (where we assumed $V_{o}^{*}=$ $10 \mathrm{eV}$ and $m_{\mathrm{eff}}=m$ ), hence in normal emission the final-state 


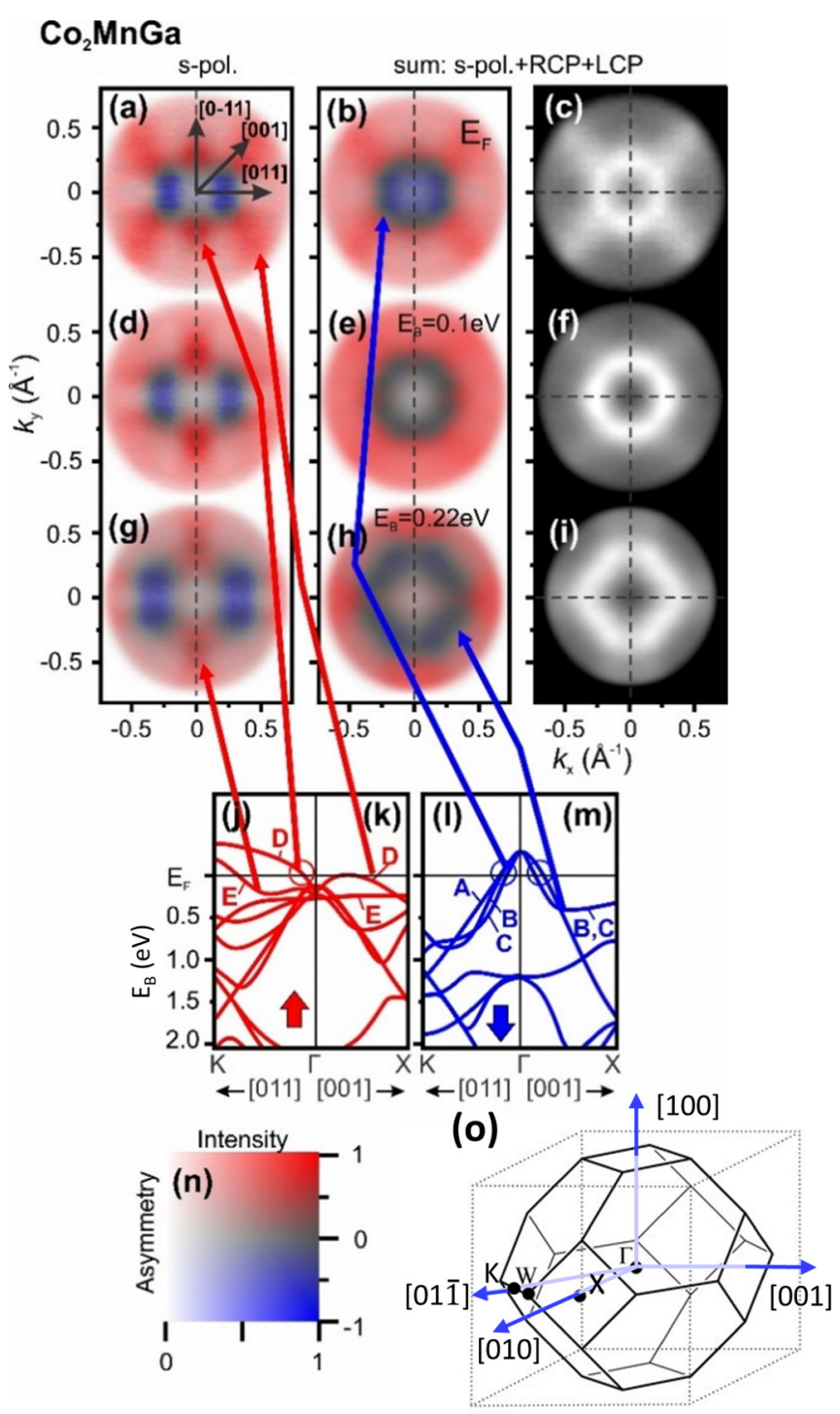

FIG. 2. $\left(k_{x}, k_{y}\right)$ momentum distributions of spin texture and intensity for in situ deposited $\mathrm{Co}_{2} \mathrm{MnGa}$ (recorded at $h v=6.05 \mathrm{eV}$ ) in comparison with theory. (a)-(c) Constant-energy sections at the Fermi energy, (d)-(f) at a binding energy of $E_{\mathrm{B}}=0.1 \mathrm{eV}$, and (g)-(i) at $E_{\mathrm{B}}=0.22 \mathrm{eV}$. The sections show the spin-asymmetry distribution of the topmost minority bands $A, B, C$ and majority bands $D$ and $E$. Distributions (a), (d), and (g) are taken with $s$-polarized light, (b), (e), (h) and (c), (f), (i) represent the sum of all three polarizations ("isotropic light"). (j),(k) Majority bands of the DFT-LSDA calculation [26] (red) and (1),(m) minority bands (blue); the energy positions of the bands have been adjusted with experiment while keeping their width constant (see original calculation results in Fig. 4). (n) 3D color code denoting majority (red), minority (blue), as well as unpolarized (grey) partial intensities. (o) Bulk and surface Brillouin zone with high-symmetry points and directions. With the lattice constant 5.7 $\AA$ the high symmetry points are at distances $\Gamma-X=\bar{\Gamma}-\bar{M}=1.1 \AA^{-1}$ and $\bar{\Gamma}-\bar{X}=0.8 \AA^{-1}$. Complete data arrays of intensity and spin texture are given as videos in the Supplemental Material [75].

sphere intersects the first repeated BZ close to the center [ $\Gamma K X$ plane in Fig. 2(o)] [72-74]. For $\mathrm{Co}_{2} \mathrm{MnGa}$, the lattice constant is larger [5.77(3) $\AA$ ] , resulting in $k_{z}=0.96 G_{100}$ for $6.05 \mathrm{eV}$. The value of $k_{z}$ significantly depends on $V_{o}^{*}$. A justification for the value assumed here for the inner potential is given in Sec. III C. Note that for high photon energies the slightly different lattice constant leads to a larger variation of $k_{z}$.

\section{RESULTS AND COMPARISON WITH CALCULATIONS}

\section{A. Identification of bands for $\mathrm{Co}_{2} \mathrm{MnGa}$}

Band features are visible most pronounced for $\mathrm{Co}_{2} \mathrm{MnGa}$, therefore we discuss these results and introduce a band labeling scheme, before we present the comparison with the two half-metallic ferromagnets in Sec. III B. For $\mathrm{Co}_{2} \mathrm{MnGa}$, we compare our results with two different types of calculations, one employing density functional theory (DFT-LSDA) and the second one dynamical mean-field theory (DMFT). The calculation from the Alabama Heusler database [26] uses the Vienna Ab-Initio Simulation Package based on a plane-wave basis set and pseudopotentials with adopted Perdew-Burke-Ernzerhof generalized gradient approximation to the exchange correlation functional. The DMFT calculation [51] was performed within the spin-polarized relativistic full-potential Korringa-Kohn-Rostoker (SPR-KKR) Green's function method. Local dynamical correlations are implemented in the DMFT calculation within SPR-KKR code in the fully self-consistent way.

Figure 2 shows $\left(k_{x}, k_{y}\right)$ sections for $\mathrm{Co}_{2} \mathrm{MnGa}$ through the spin-texture array at $E_{\mathrm{F}}$ for $s$-polarized light (a) and for the sum of all three light polarizations (b). The intensity pattern for the sum is shown in (c). The second and third rows show the analogous results at binding energies of $E_{\mathrm{B}}=0.1 \mathrm{eV}[(\mathrm{d})-$ (f)] and $0.22 \mathrm{eV}[(\mathrm{g})-(\mathrm{i})]$. The outer diameter of the observed $k$ field is reduced with increasing binding energy because the photoemission horizon shrinks with reduced kinetic energy [Fig. 1(b)]. For comparison, the DFT-LSDA calculation along the directions $\Gamma-K$ and $\Gamma-X$ is shown for majority [(j) and (k)] and minority bands [(l) and (m)]. These directions correspond to the horizontal axis $k_{x}$ and the diagonal, as denoted in Fig. 2(a). The absolute energy positions of the calculated bands deviate from the measured positions. For the sake of clarity, the theoretical bands have been individually shifted to align with experiment (details in Sec. IV A).

The three-dimensional color code of the spin-resolved patterns is defined in Fig. 2(n): Red and blue denote the partial majority- and minority-spin intensities, whereas the neutral grey value quantifies unpolarized intensity. In this code, unpolarized bands or background show up in the displayed spin texture (whereas in pure red-blue encoding these would be missing). A blue band on a homogeneous red background may appear grey if the sum of minority and majority spins just compensates each other. Such a band can be emphasized, however, if a homogeneous majority background is subtracted. This is a special advantage of the 3D spin-recording architecture.

The bulk and surface Brillouin zones are shown in Fig. 2(o), which defines the directions in 3D $k$ space. Given the surface normal [100], the high-symmetry directions in our observed $k$ field are [011] (horizontal), [0-11] (vertical), and [001] as well as [010] (both diagonal). The pronounced spin texture of the momentum pattern at $E_{\mathrm{F}}$ [Figs. 2(a) and 2(b)] reveals several details. The eye-catching features are two 
deep-blue bars (a), which are completed to form a blue ring in the sum pattern (b). Due to the cubic symmetry, directions [011] and [0-11] (horizontal and vertical) are equivalent and the band features in the ground state are identical. However, the photoemission process with $s$ - or $p$-polarized light breaks the symmetry, leading to emission from different initial bands due to the symmetry selection rules. Although $k_{x}$ and $k_{y}$ are equivalent concerning the ground-state wave functions, the $s$-polarized excitation (electric vector along $k_{y}$ ) emphasizes different bands due to the symmetry selection rules, patterns (a), (d), and (g). Figures 2(b) and 2(c), derived from the sum arrays for $s$-polarized, RCP, and LCP (equivalent to "isotropic light"), are essentially free from dichroism effects. A red cross is oriented along the [001] and [010] directions (diagonal). Red dots are visible in (a) above and below the image center, which develop into a red ring surrounding the blue ring in (b). With increasing binding energy the blue bars disperse outwards [sequence (a), (d), (g)], the red cross fades out, and two vertical red bars appear in $(\mathrm{g})$. In the intensity patterns [sequence (c), (f), (i)] the bright circle close to the center (c) first expands (f) and then develops into a diamond-shaped feature (i). The diagonal cross reduces strongly in intensity within the first $100 \mathrm{meV}$ below $E_{\mathrm{F}}$ [compare (c) and (f)].

Inspection of the DFT-LSDA calculation for $\mathrm{Co}_{2} \mathrm{MnGa}$ [Figs. 2(j)-2(m)] reveals that all observed band features and their polarization signature are clearly related to the calculated valence bands of bulk $\mathrm{Co}_{2} \mathrm{MnGa}$. The binding energies of individual band groups, however, deviate from the experimental result at $\Gamma$.

We label the bands by capital letters sorted with increasing binding energy along $\Gamma-K$. The topmost bands close to $E_{\mathrm{F}}$ are three minority bands $A, B$, and $C$ (blue), degenerate at $\Gamma$ slightly above $E_{\mathrm{F}}$, which split into two branches along $\Gamma-X$ and three branches along $\Gamma-K$ (having lower symmetry than $\Gamma-X)$. According to the calculation these bands disperse similarly in all directions, calculation in Figs. 2(l) and 2(m) (see [26] for the $\Gamma-L$ direction). Experimentally they appear as an expanding blue ring close to $E_{\mathrm{F}}$, Figs. 2(b) and 2(e) (left blue arrow). The topmost majority bands $D$ and $E$ close to $E_{\mathrm{F}}$ both disperse upwards with increasing $k_{\|}$, but highly anisotropic; see calculation in Figs. 2(j) and 2(k). Band $D$ crosses $E_{\mathrm{F}}$ at some distance from the $\Gamma$ point along the $\Gamma-K$ direction [red dot in (a), middle red arrow], whereas it shows a broad maximum along the $\Gamma-X$ direction [intensity cross in (c) with red spin signature (a) along the diagonal directions [001] and [010], denoted by the right red arrow]. The second majority band $E$ shows a shallow minimum along $\Gamma-K$ [Fig. 2(j)], which is experimentally visible as vertical red bars in Fig. 2(g) (denoted by the left red arrow). Band $E$ crosses the Fermi level close to the $K$ point, which lies outside of the visible horizon at $h v=6.05 \mathrm{eV}$.

The shallow band $D$ (red cross in diagonal orientation) disappears already $100 \mathrm{meV}$ below $E_{\mathrm{F}}$, hence its maximum exactly coincides with the Fermi edge. Between Figs. 2(d) and 2(e) and Figs. 2(g) and 2(h) the diagonal region changes from red to grey, indicating the presence of the horizontal branch of the degenerate minority bands $B$ and $C$ in the $\Gamma-X$ direction (right blue arrow). A blue band on a red background appears in grey. The center of all patterns exhibits a rather low intensity, which we attribute to a matrix-element effect at $h v=6.05 \mathrm{eV}$.
The obvious deviation from the fourfold crystal symmetry in the photoelectron spin texture for $s$-polarized excitation (first column of Fig. 2) is a consequence of the symmetry selection rules. The dipole operator for polarized light selectively excites partial waves with a certain value of orbital quantum number $l \rightarrow l \pm 1$ (common dipole selection rule) and additionally selects certain quantum numbers $m_{1}$. The same effect causes the linear dichroism (dissymmetry) in the photoelectron angular distribution (LDAD) for $s$-polarized light (here electric vector along [0-11]). Such LDAD asymmetries appear very clearly in $k$-microscope images as observed in our previous work using Synchrotron radiation [62]. The selectivity of these symmetry selection rules can be exploited for the separation and identification of bands with different spin signature that are partly overlapping. The blue and red features are completely separated in Figs. 2(a), 2(d), and 2(g), whereas they are overlapping in Figs. 2(b), 2(e), and 2(h), even partly canceling each other in (e). On the other hand, the almost circular cross section of bands $A-C$ shows up only in the sum images (b), (e), and (h). It means that both representations have their specific advantages. Videos of the complete data arrays of Figs. 2(a)-2(c) and a more detailed comparison with calculations are given in the Supplemental Material [75].

The dependence of photoemission intensity on the polarization of incident light contains information on the orbital character of initial states near $\Gamma$ : The prevailing intensity of minority bands $A-C$ along $k_{x}$ in Figs. 2(a), 2(d), and 2(g) indicates the $d_{x z}$ character of the highest occupied minority state. The intensity of majority states outreaches the minority intensity along $k_{y}$. This is explained by the $d_{z^{2}}$ and $d_{x^{2}-y^{2}}$ character of bands $D$ and $E$ near $E_{\mathrm{F}}$ leading to a high photoemission intensity along $k_{y}$ for $s$ polarization. We conclude that $\mathrm{Co}_{2} \mathrm{MnGa}$ is not half metallic because both majority (red) and minority bands (blue) cross the Fermi surface. In particular, there is an intense outward-dispersing minority band which has a circular shape in the $\left(k_{x}, k_{y}\right)$ sections [Figs. 2(b), 2(e), and 2(h)], because it crosses the Fermi level in a region with almost isotropic dispersion.

\section{B. Comparison of all three Heusler compounds}

Figure 3 shows an overview of results recorded for all three compounds using $s$-polarized light (suppressing surface-state emission [21]). Figures 3(a)-3(c) show the $\left(k_{x}, k_{y}\right)$ intensity distributions at the Fermi energy for $\mathrm{Co}_{2} \mathrm{MnGa}, \mathrm{Co}_{2} \mathrm{MnSi}$, and $\mathrm{Co}_{2} \mathrm{Fe}_{0.4} \mathrm{Mn}_{0.6} \mathrm{Si}$. In contrast to the pronounced band features for $\mathrm{Co}_{2} \mathrm{MnGa}$ (a), both Si-based compounds exhibit a homogeneous low-intensity grey level without any indication of bands (b) and (c). The top of the first band for $\mathrm{Co}_{2} \mathrm{Fe}_{0.4} \mathrm{Mn}_{0.6} \mathrm{Si}$ appears as a central dot at a binding energy of $0.3 \mathrm{eV}$ (d) and develops into a pattern of two vertical bars and two dots above and below the center at $E_{\mathrm{B}}=0.5 \mathrm{eV}(\mathrm{h})$. This pattern looks similar to the central part of the distribution for $\mathrm{Co}_{2} \mathrm{MnGa}$ at $E_{\mathrm{F}}$ (a). The corresponding pattern for $\mathrm{Co}_{2} \mathrm{MnSi}$ at $E_{\mathrm{B}}=0.7 \mathrm{eV}$ (l) also shows two vertical bars at the left and right rim of the $k$ field of view. The $E_{\mathrm{B}}-\mathrm{vs}-k_{x}[(\mathrm{e})-$ $(\mathrm{g})]$ and $E_{\mathrm{B}}-\mathrm{vs}-k_{y}$ sections [(i)-(k)] show the band dispersions along the [011] and [0-11] directions. The work functions are higher for the Si-containing compounds $(4.6 \pm 0.1 \mathrm{eV}$ for 


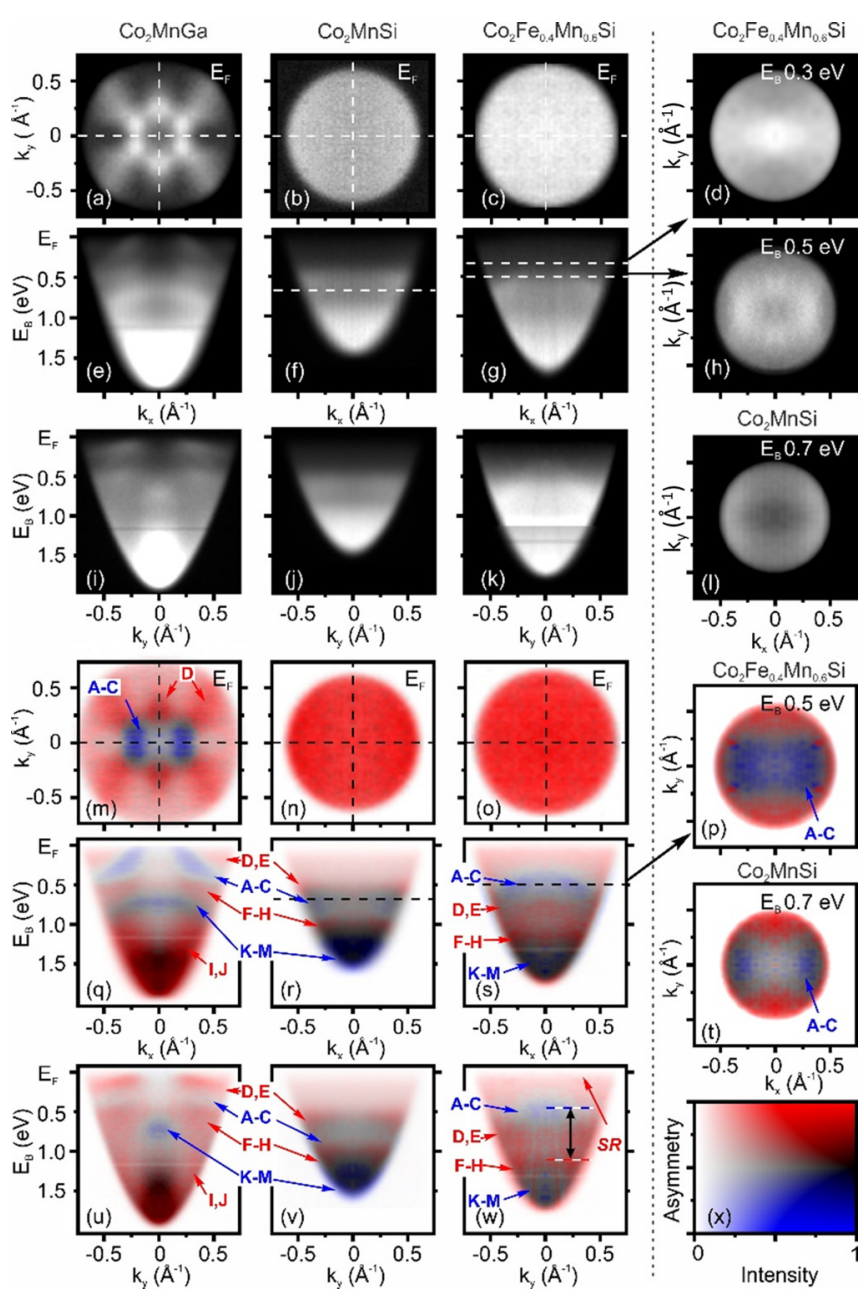

FIG. 3. Comparison of the spin-resolved valence-band structure of in situ deposited $\mathrm{Co}_{2} \mathrm{MnGa}, \mathrm{Co}_{2} \mathrm{MnSi}$, and $\mathrm{Co}_{2} \mathrm{Fe}_{0.4} \mathrm{Mn}_{0.6} \mathrm{Si}$ films. Spin-integral (a)-(l) and spin-resolved sections (m)-(w) are shown along the indicated cuts through the data arrays (thin dashed lines). All results measured using $s$-polarized light $(h v=6.05 \mathrm{eV})$ with electric vector along $k_{y}$. The $\left(k_{x}, k_{y}\right)$ sections at the Fermi energy (a)-(c) and at binding energies of $E_{\mathrm{B}}=0.3 \mathrm{eV}(\mathrm{d}), 0.5 \mathrm{eV}(\mathrm{h})$, and $0.7 \mathrm{eV}$ (l) show the momentum distributions of the intensity. The corresponding spin textures are shown in $(\mathrm{m})-(\mathrm{p}),(\mathrm{t})$, and $(\mathrm{x})$. Band dispersions and their spin texture are visible in the $E_{\mathrm{B}}-\mathrm{vs}-k_{x}(\mathrm{e})-(\mathrm{g})$ and (q) $-(\mathrm{s})$ and $E_{\mathrm{B}}-\mathrm{vs}-k_{y}$ sections (i) $-(\mathrm{k})$ and (u)-(w). Labels $A-M$ denote equivalent bands in the three compounds. 3D color code like in Fig. 2(n). In (r)-(t), (v), and (w) a majority background is subtracted by shifting the zero point of the color code and the color scale is stretched, in order to emphasize the blue bands. In $(\mathrm{k})$ the image contrast in the upper part is increased to make the top of the band visible. In (w) the black double arrow marks an exchange-split pair of band groups $A-C$ and $F-H$. SR in (w) denotes a weak signature of a surface resonance close to $E_{\mathrm{F}}$.

$\mathrm{Co}_{2} \mathrm{MnSi}$ and $4.5 \pm 0.1 \mathrm{eV}$ for $\mathrm{Co}_{2} \mathrm{Fe}_{0.4} \mathrm{Mn}_{0.6} \mathrm{Si}$ ) than for $\mathrm{Co}_{2} \mathrm{MnGa}(4.1 \pm 0.1 \mathrm{eV})$. This reduces the observed diameter in $k$ space because the bottoms of the paraboloids in the second and third columns of Fig. 3 are shifted upwards.

The spin character of the observed bands is revealed in Figs. 3(m)-3(w). $k_{x}$ and $k_{y}$ are equivalent concerning the ground-state wave functions, but the $s$-polarized exci-
TABLE I. Experimental band positions (binding energies) and minority gaps with respect to $E_{\mathrm{F}}$. The negative value corresponds to a position above $E_{\mathrm{F}}$ as estimated from comparison to calculations. The gap values denote the part of the spin gap in the occupied band structure. Last row, measured exchange splitting of band groups $A-C$ and $F-H$ at the $\Gamma$ point of the Brillouin zone.

\begin{tabular}{lccc}
\hline \hline Band & Position $(\mathrm{eV}) \mathrm{Co}_{2} \mathrm{MnGa}$ & $\mathrm{Co}_{2} \mathrm{MnSi}$ & $\mathrm{Co}_{2} \mathrm{Fe}_{0.4} \mathrm{Mn}_{0.6} \mathrm{Si}$ \\
\hline$A-C \downarrow$ & $-0.15^{\mathrm{a}}$ & 0.5 & 0.35 \\
$K-M \downarrow$ & 0.71 & 1.4 & 1.5 \\
$D-E \uparrow$ & 0 & 0.6 & 0.74 \\
$F-H \uparrow$ & 0.33 & 1.05 & 1.1 \\
$I-J \uparrow$ & 1.1 & & \\
$\downarrow$ gap & & $0.5 \pm 0.1$ & $0.35 \pm 0.05$ \\
$\Delta E_{\text {ex }}$ & $0.48 \pm 0.07$ & $0.55 \pm 0.10$ & $0.72 \pm 0.07$ \\
\hline \hline
\end{tabular}

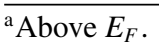

tation (electric vector along $k_{y}$ ) emphasizes different bands due to the symmetry selection rules. The $E_{\mathrm{B}}$-vs- $k_{x}$ sections, Figs. 3(q)-3(s), emphasize different bands than the $E_{\mathrm{B}}-\mathrm{vs}-k_{y}$ sections $[(\mathrm{u})-(\mathrm{w})]$, as is clearly visible in the spin texture. The $\left(k_{x}, k_{y}\right)$ distributions at $E_{\mathrm{F}}[(\mathrm{m})-(\mathrm{o})]$ exhibit the striking difference between $\mathrm{Co}_{2} \mathrm{MnGa}$ with red and blue bands crossing $E_{\mathrm{F}}$ (m) and the two half-metallic ferromagnets showing a uniform bright-red color, which is characteristic for pure majority-spin density, Figs. 3(n) and 3(o). This uniform red color extends from $E_{\mathrm{F}}$ to $E_{\mathrm{B}} \approx 0.3 \mathrm{eV}$; see videos in the Supplemental Material [75]. We briefly summarize the results for the three compounds; equivalent bands are labeled with the same letters as introduced for $\mathrm{Co}_{2} \mathrm{MnGa}$. Table I summarizes measured band positions, minority gaps, and exchange splitting values.

For $\mathrm{Co}_{2} \mathrm{MnGa}$ (first column) we determine an exchange splitting of $\Delta E_{\mathrm{ex}}=0.48 \pm 0.07 \mathrm{eV}$ between the frontier band groups $A-C$ and $F-H$ at the $\Gamma$ point. The top of the minority band group $A-C$ lies at $\sim 0.2 \mathrm{eV}$ above $E_{\mathrm{F}}$, as estimated by comparison of the occupied part with calculated band dispersion; i.e., there is no spin gap, in agreement with [21].

For $\mathrm{Co}_{2} \mathrm{MnSi}$ (second column) the band features appear more diffuse and the central part of minority bands $A-C$ is obscured by the overlapping majority bands $D-E$ appearing at the same binding energy of $\sim 0.6 \mathrm{eV}$ [Figs. 3(r) and 3(v)]. In order to emphasize the blue band on a red background, a constant majority background is subtracted and the color scale of Figs. 3(r) and 3(v) is stretched. The outer region of $A-C$ is visible as vertical blue bars in the $\left(k_{x}, k_{y}\right)$ section Fig. 3(x), taken along the dashed line in (r). The similarity with the blue bars in Fig. $3(\mathrm{~m})$ for $\mathrm{Co}_{2} \mathrm{MnGa}$ is obvious. The maximum of bands $A-C$ in $\mathrm{Co}_{2} \mathrm{MnSi}$ can be located at $E_{\mathrm{B}} \approx 0.50 \mathrm{eV}$, defining a minority spin gap (with respect to $\left.E_{\mathrm{F}}\right)$ of $0.5 \pm 0.1 \mathrm{eV}$. This value is in perfect agreement with the spin-DOS calculation in Ref. [34] and the measurement with $s$-polarization by Guillemard et al. (Fig. 2 of Ref. [21]). Majority band group $F-H$ is visible at $\sim 1.05 \mathrm{eV}$ and minority group $K-M$ appears at $\sim 1.4 \mathrm{eV}$ close to the bottom of the paraboloid. The exchange splitting of band groups $A-C$ and $F-H$ at $\Gamma$ is $\Delta E_{\text {ex }}=0.55 \pm 0.10 \mathrm{eV}$.

For $\mathrm{Co}_{2} \mathrm{Fe}_{0.4} \mathrm{Mn}_{0.6} \mathrm{Si}$ (third column) the top of the first minority band (blue) is not obscured and clearly visible at 

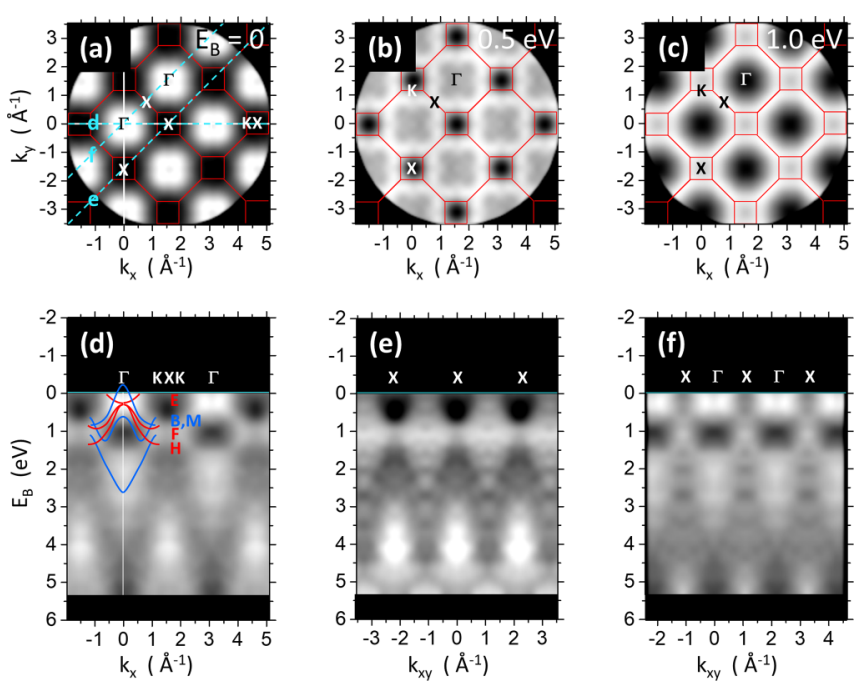

FIG. 4. Valence-band structure of a $\mathrm{Co}_{2} \mathrm{MnGa}$ thin film measured at a photon energy of $3438 \mathrm{eV}$. The $\left(k_{x}, k_{y}\right)$ sections at the Fermi energy (a) and at binding energies of $E_{\mathrm{B}}=0.5 \mathrm{eV}$ (b), and $1.0 \mathrm{eV}$ (c) show the transversal momentum distributions of the intensity. Band dispersions are visible in the $E_{\mathrm{B}}-\mathrm{vs}-k_{x}(\mathrm{~d})$ and $E_{\mathrm{B}}-\mathrm{vs}-k_{x y}$ sections (e),(f) along the traces indicated in (a). Blue (red) lines in (d) indicate adapted band dispersions of minority (majority) states from a DFT-LSDA calculation [26] [see Figs. 6(g) and 6(h)].

$E_{\mathrm{B}} \approx 0.35 \mathrm{eV}$ in the $E_{\mathrm{B}}-\mathrm{vs}-k_{x}$ and $-k_{y}$ sections [Figs. 3(s) and $3(\mathrm{w})]$. Intensity $\left(k_{x}, k_{y}\right)$ sections (d) and (h) and spin patterns in $(\mathrm{p})$ and $(\mathrm{t})$ clearly prove that the top of the minority band lies significantly higher than in $\mathrm{Co}_{2} \mathrm{MnSi}$ [(1) and (t)]. Figure 3(p) shows the spin-polarization pattern corresponding to the intensity pattern (d), revealing the appearance of the top of the minority band (blue) in the center. The minorityspin gap (measured with respect to $E_{\mathrm{F}}$ ) is $0.35 \pm 0.05 \mathrm{eV}$, significantly smaller than for $\mathrm{Co}_{2} \mathrm{MnSi}$. Majority bands $D-E$ are well separated from bands $A-C$. The second majority band group $F-H$ is clearly visible as red band with maximum at $1.07 \pm 0.05 \mathrm{eV}$ in Figs. 3(s) and 3(w). The exchange splitting of bands $A-C$ and $F-H$ at $\Gamma$ is $\Delta E_{\mathrm{ex}}=0.72 \pm 0.07 \mathrm{eV}$ [denoted by the dashed lines and black double-arrow in Fig. 3(w)]. The second minority band group $K-M$ is visible at $\sim 1.5 \mathrm{eV}$ close to the bottom of the photoemission paraboloid. Only a very weak trace of a possible surface resonance (SR) for $\mathrm{Co}_{2} \mathrm{Fe}_{0.4} \mathrm{Mn}_{0.6} \mathrm{Si}$ is visible in Fig. $3(\mathrm{w})$ close to $E_{\mathrm{F}}$, in analogy to the resonance observed for $\mathrm{Co}_{2} \mathrm{MnSi}$ [34].

\section{Bulk bands of $\mathrm{Co}_{2} \mathrm{MnGa}$ and $\mathrm{Co}_{2} \mathrm{MnSi}$ observed using hard $x$ rays}

In order to complement the low-energy results in a larger $k$ range comprising several Brillouin zones, we investigated $\mathrm{Co}_{2} \mathrm{MnGa}$ and $\mathrm{Co}_{2} \mathrm{MnSi}$ at photon energies in the hard $\mathrm{x}$-ray regime using the same technique, however without spin filter. Uncapped films fabricated under identical conditions as for the $6.05-\mathrm{eV}$ measurements have been transported from the lab in Mainz to PETRA III (Hamburg) using a UHV suitcase. Figures 4 and 5 show data measured at photon energies of $3300 \mathrm{eV}\left(\mathrm{Co}_{2} \mathrm{MnSi}\right)$ and $3438 \mathrm{eV}\left(\mathrm{Co}_{2} \mathrm{MnGa}\right)$, respectively, corresponding to a perpendicular momentum of (see [72]) $k_{\mathrm{z}}=13 G_{001}$ for $\mathrm{Co}_{2} \mathrm{MnSi}$ and $\mathrm{Co}_{2} \mathrm{MnGa}$. At these high pho-
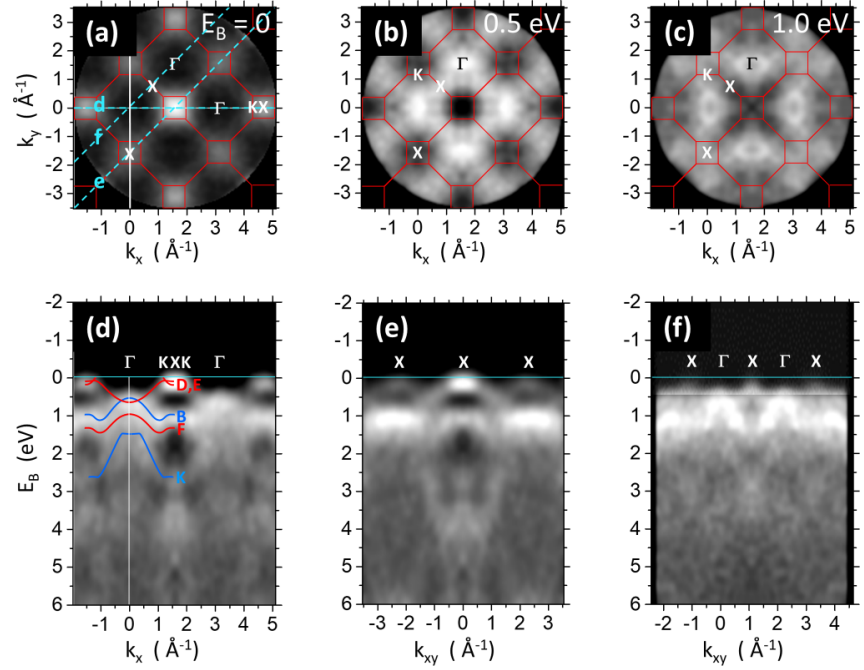

FIG. 5. Valence-band structure of a $\mathrm{Co}_{2} \mathrm{MnSi}$ thin film measured at a photon energy of $3300 \mathrm{eV}$. The $\left(k_{x}, k_{y}\right)$ sections at the Fermi energy (a) and at binding energies of $E_{\mathrm{B}}=0.5 \mathrm{eV}$ (b) and $1.0 \mathrm{eV}$ (c) show the momentum distributions of the intensity. Band dispersions are visible in the $E_{\mathrm{B}}-\mathrm{vs}-k_{x}(\mathrm{~d})$ and $E_{\mathrm{B}}$-vs- $k_{x y}$ sections (e),(f). Blue (red) lines in (d) indicate adapted band dispersions of minority (majority) states from a DFT-LSDA calculation [26] [see Figs. 6(i) and 6(j)].

ton energies, the effective mass equals the free-electron mass and the perpendicular momentum is insensitive to the assumed value of the inner potential. In reciprocal space, the transition at this energy probes a flat section through the $\Gamma-K-X$ plane [see Fig. 2(o)] in the 13th repeated Brillouin zone along $k_{z}$. A substantial contribution of photoelectron diffraction, typical for high-energy angular resolved photoemission spectroscopy (HARPES), had to be eliminated in the data shown in Figs. 4 and 5; for details, see [72,73]. The energy resolution is determined by the bandwidth of the monochromator $(340 \mathrm{meV})$. Due to the drop of photoemission cross sections [74] spinresolved measurements were not possible at this energy.

For $\mathrm{Co}_{2} \mathrm{MnGa}$, the $\left(k_{x}, k_{y}\right)$ section at $E_{\mathrm{F}}$ [Fig. 4(a)] reveals a fourfold-symmetric bright area with rounded corners centered at the $\Gamma$ point. The regions around the $X$ and $K$ points (closely spaced in this cut through the periodic array of Brillouin zones in 3D $k$ space) appear dark at $E_{\mathrm{F}}$. With increasing binding energy, this bright intensity develops into a ring with increasing diameter. At $E_{\mathrm{B}}=0.5 \mathrm{eV}$ [Fig. 4(b)], the rings of adjacent $\Gamma$ points touch each other at the $X$ point. With further increasing $E_{\mathrm{B}}$, the center of the ring at $\Gamma$ turns dark [Fig. 4(c)], pointing on a local band gap. The band dispersions along three high-symmetry directions depicted in Figs. 4(d)-4(f) correspond to the dashed lines in (a). Indeed, the $E_{\mathrm{B}}-\mathrm{vs}-k_{x}$ and $-k_{x y}$ sections reveal the local gaps in the $K X K$ region at $E_{\mathrm{F}}$ [see (d) and (e)] and around the $\Gamma$ point at $E_{\mathrm{B}}=1 \mathrm{eV}$ [see (d) and (f)]. Gap positions and dispersions of the band groups are in good agreement with the DFT-LSDA calculation [26] with the adapted majority and minority bands, shown as blue and red curves in Fig. 4(d). The bright region at $E_{\mathrm{F}}$ (a) and expanding ring in (b) and (c) can thus be assigned to the downward-dispersing minority band $B$ with an intensity 


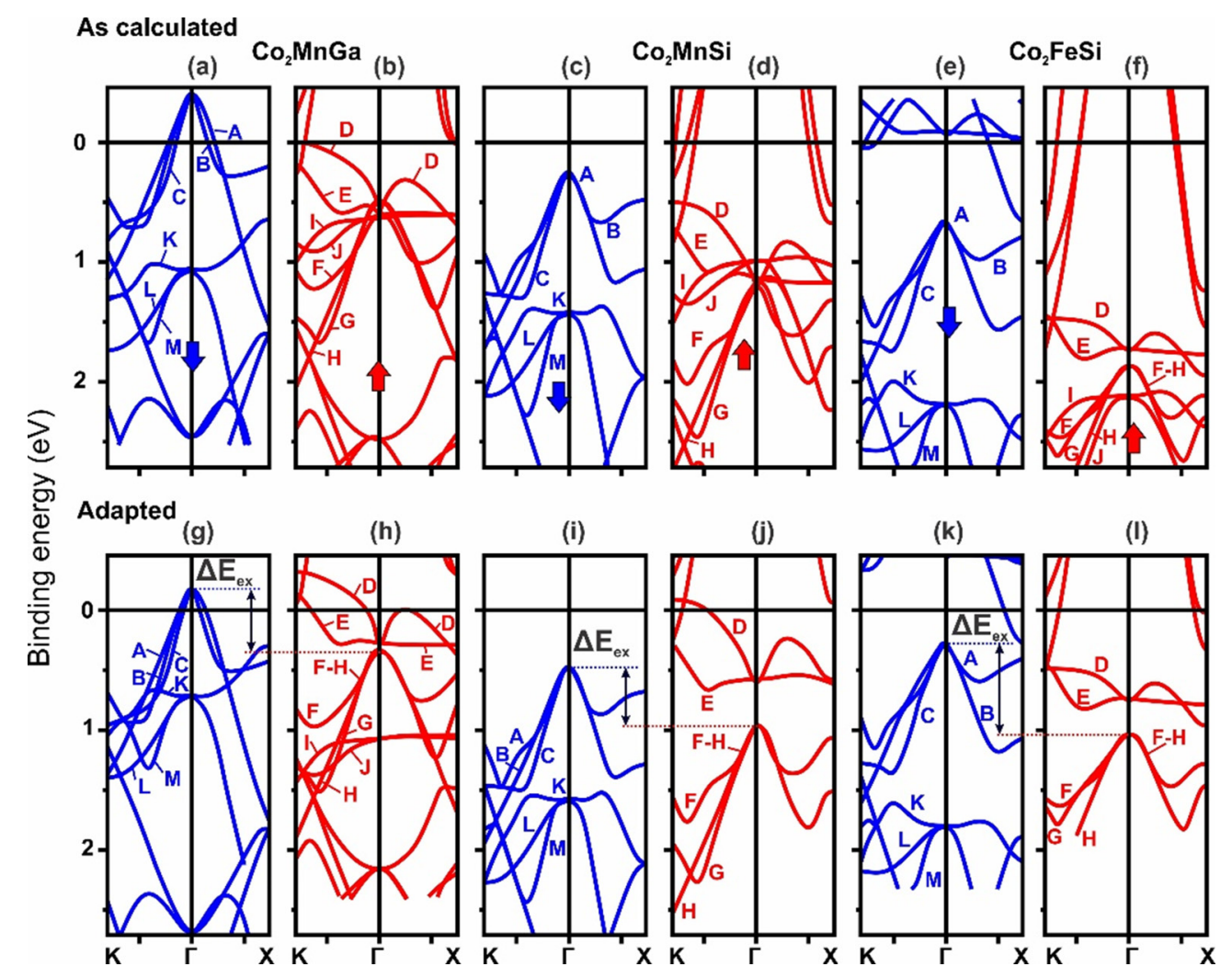

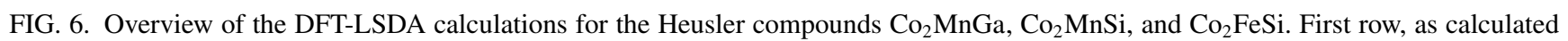
(from [26]); second row, adjusted to the experimental band positions. The deficiency of majority band electrons causes an upward shift of the calculated majority bands [compare (b), (d), and (f) with (h), (j), and (l)]. The measured exchange splitting values $\Delta E_{\text {ex }}$ between bands $A$ - $C$ and $F-H$ of $0.48,0.55$, and $0.72 \mathrm{eV}$ are marked by red and blue dotted lines in (g)-(l). Since no calculation exists for $\mathrm{Co}_{2} \mathrm{Fe}_{0.4} \mathrm{Mn}_{0.6} \mathrm{Si}_{1}$ the results for this compound are compared with the calculated bands for $\mathrm{Co}_{2} \mathrm{FeSi}$ with an interpolated Fermi level.

contribution from majority band E. Figure 4(c) reveals the energy gap at $\Gamma$ at a binding energy of $1 \mathrm{eV}$.

The band dispersions measured at $h v=3438 \mathrm{eV}$ clearly agree with the adapted band positions and with the photoemission results obtained with 6.05-eV excitation. Despite the limited resolution of the HARPES experiment, we find a fair agreement with the low-energy results near the $\Gamma$ point as shown in Fig. 3, first column. The high intensity of the region close to the $X$ points in (d)-(f) at $E_{\mathrm{B}}=1 \mathrm{eV}$ indicates a high density of states due to the overlap of several majority and minority bands $(B, M, F, H)$ with a small slope, as predicted by the calculation shown in Fig. $6(\mathrm{~g})$.

In the case of $\mathrm{Co}_{2} \mathrm{MnSi}$, the $\left(k_{x}, k_{y}\right)$ section at the Fermi energy [Fig. 5(a)] and the $E_{\mathrm{B}}$-vs- $k_{x}$ section (d) reveal the minority band gap, visible as a dark area without any band features near the $\Gamma$ point, in accordance with the results in Fig. 3 (second column). Areas of high intensity appear close to the $X$ and $K$ points in the Fermi-energy section (a). In the $6.05-\mathrm{eV}$ measurements, these features lie outside of the photoemission horizon. Hence the measurement at $3300 \mathrm{eV}$ nicely complements the low-energy results. At a binding energy of $0.5 \mathrm{eV}$ [Fig. 5(b)] a spot of high intensity appears at the $\Gamma$ point and in Figs. 5(d) and 5(f) the corresponding band with its maximum at $0.5 \mathrm{eV}$ at $\Gamma$ shows a downward dispersion. This signal can thus be assigned to the top of the minority band group $A-C$, in perfect agreement with the low-energy data shown in Fig. 3. As anticipated, the spot of high intensity opens up to a round band feature at higher binding energy as visible in Fig. 5(c), indicating a negative group velocity of this minority band with similar velocities along $\Gamma-X$ and $\Gamma-K$. The intensity asymmetry at $\Gamma\left(k_{x}=0\right)$ along $k_{x}$ stems from a photoemission contribution of higher harmonic photons appearing at all four $\Gamma$ points due to the fourfold symmetrization.

The band dispersions along three high-symmetry directions depicted in Figs. 5(d)-5(f) correspond to the dashed lines in (a). In Fig. 5(d) the measured $\Gamma-K-X$ band dispersion has been overlaid with the adapted majority and minority bands from the DFT-LSDA calculation [26] [Figs. 6(i) and 6(j)]. Taking into account the limited energy resolution, we find very good agreement with the low-energy results at the $\Gamma$ point. In addition, we now observe the band dispersion within the complete Brillouin zone. This allows for a more precise location of the majority band $D$ and confirms that it crosses (or touches) $E_{\mathrm{F}}$ close to the $K$ point, as predicted by theory [Fig. 6(j)]. The high intensity of the region close to the $K$ points in (d) indicates a high density of states due to a vanishing slope of band $D$ close to the Fermi edge, as predicted by the calculation in Fig. 6(j).

For the determination of the inner potential in Eq. (2), we take the photoemission spectra obtained at high photon energy as a reference. The agreement of the valence band energies near the $\Gamma$ point as measured by HARPES and by $6.05-\mathrm{eV}$ excitation suggests that both experiments probe planes with a similar $k_{z}$ value (modulo $G_{100}$ ). This comparison is sen- 
sitive on $k_{z}$, because the position of the strongly dispersive minority valence band $B$ strongly depends on $k_{z}$. In the case of HARPES, $k_{z}$ results from assuming a free-electron-like final state and no assumption on the inner potential is necessary [72]. Furthermore, we verified the probing of the $\Gamma-K-X$ plane by varying the photon energy. For $6.05-\mathrm{eV}$ excitation, the inner potential significantly varies the probed $k_{z}$ value. The coincidence of the band energies justifies our assumption (approximately $10 \mathrm{eV}$ ) on the inner potential within error margins. For the error discussion, we state that the radius $0.22 \AA^{-1}$ of the ring formed by minority band $B$ at $E_{\mathrm{F}}$ for the case of $\mathrm{Co}_{2} \mathrm{MnGa}$ [Fig. 2(c)] provides a lower limit of the unoccupied maximum of this band at $\Gamma$. Using the measured velocity in the transversal $\Gamma-X$ direction [Fig. 3(q)], one obtains a band maximum of $(0.20 \pm 0.05) \mathrm{eV}$. This value provides a lower limit because an actual deviation from the $\Gamma$ point along $\pm k_{\mathrm{z}}$ for the $6.05-\mathrm{eV}$ excitation will result in a smaller ring diameter. Hence, the diameter and the band maximum are largest for $k_{z}=G_{100}$. The upper limit is defined by the presence of the band gap at $\Gamma$ between bands $M$ and I at approximately $1 \mathrm{eV}$ [Figs. 3(e) and 3(q)], which would close for a deviation from $k_{z}=G_{100}$ by more than $\pm 0.2 \AA^{-1}$ as revealed by the HARPES data shown in Fig. 4(d). Such a deviation for 6.05$\mathrm{eV}$ excitation would shift the band maximum to higher energy by approximately $0.2 \mathrm{eV}$. For $\mathrm{Co}_{2} \mathrm{MnGa}$ we thus arrive at a band maximum of $\left(E_{\mathrm{F}}+0.20_{-0.05}^{+0.20}\right) \mathrm{eV}$.

\section{DISCUSSION}

Following the well-known 24-electron rule for full-Heusler compounds, their electronic structure is determined by the number of valence electrons [76]. In a simple rigid-band model, electrons tend to fill unoccupied band states with increasing number of valence electrons without changing the band dispersions. In a more atomiclike view the most electropositive element will give its electron to fill the open shells of more electronegative elements within the compound [1]. According to group theory, the minority band of a half-metallic ferromagnetic full-Heusler compound contains exactly 12 electrons [24]. The full-Heusler compounds show a Slater-Pauling behavior and the total spin-magnetic moment per unit cell $m$ scales with the total number of valence electrons $N_{v}$ following the rule $m\left(N_{v}\right)=N_{v}-24$. Thus, $\mathrm{Co}_{2} \mathrm{MnGa}, \mathrm{Co}_{2} \mathrm{MnSi}$, and $\mathrm{Co}_{2} \mathrm{Fe}_{0.4} \mathrm{Mn}_{0.6} \mathrm{Si}$ would have an excess electron number of 4,5 , and 5.4 electrons and thus show magnetic moments of 4,5 , and $5.4 \mu_{\mathrm{B}}$ per formula unit, respectively.

The degree of band filling is the key factor for the different properties of this family of Heusler compounds. Most importantly, the band filling decides whether a material is a half-metallic ferromagnet and determines the size of the gap in the minority-spin channel. Along with the aspect of probing the Heusler band structure in general, the central goal of the present work is to determine the positions of the Fermi level in both spin channels of the three compounds with different numbers of valence electrons and compare the results with theoretical predictions. Besides the different numbers of valence electrons the strength of correlation effects is expected to be different for the three compounds. Here we compare the measured band positions of all three compounds with DFT-LSDA calculations (Sec. IV A) for $\mathrm{Co}_{2} \mathrm{MnSi}$ with ten different theoretical approximations (Sec. IV B) and with existing experimental results from earlier work in literature (Sec. IV C).

Note that electron correlations not only influence the binding energy but also the width of the electronic bands. DMFT calculations for ferromagnets predict a broadening of the spectral function because of their partially filled $d$ states, which is due to electron-hole excitations [77]. This has been explicitly shown for $\mathrm{Co}_{2} \mathrm{MnSi}[30,31]$. Therefore, the broadened band dispersions observed here are partially due to electron correlation effects. The HARPES results (see Fig. 5) reveal that in the case of $\mathrm{Co}_{2} \mathrm{MnSi}$ no band states occur near $\Gamma$ in an energy interval from the Fermi level to a binding energy of $0.5 \mathrm{eV}$. For $6.05-\mathrm{eV}$ excitation the momentum field of view decreases with increasing binding energy [see photoemission paraboloid in Fig. 1(b)], preventing a clear identification of band dispersions. The same restriction applies for $\mathrm{Co}_{2} \mathrm{Fe}_{0.4} \mathrm{Mn}_{0.6} \mathrm{Si}$. Moreover, in this case the statistical distribution of $\mathrm{Fe}$ and $\mathrm{Mn}$ atoms might lead to an additional broadening.

\section{A. Band positions in comparison with DFT-LSDA calculations}

Figure 6 shows the DFT-LSDA calculations of Ma et al. (Alabama database) [26] for the bulk bands of $\mathrm{Co}_{2} \mathrm{MnGa}$, $\mathrm{Co}_{2} \mathrm{MnSi}$, and $\mathrm{Co}_{2} \mathrm{FeSi}$. The latter compound is used for the comparison with $\mathrm{Co}_{2} \mathrm{Fe}_{0.4} \mathrm{Mn}_{0.6} \mathrm{Si}$, keeping in mind that $\mathrm{Co}_{2} \mathrm{FeSi}$ has 6 (instead of 5.4) valence electrons per formula unit. Since $\mathrm{Co}_{2} \mathrm{FeSi}$ crystallizes mainly in the $B 2$ modification, we instead studied the stable $L 2_{1}$ phase of $\mathrm{Co}_{2} \mathrm{Fe}_{0.4} \mathrm{Mn}_{0.6} \mathrm{Si}$ (Fe and $\mathrm{Mn}$ share the same lattice sites). For the comparison we interpolate the position of the Fermi level between $\mathrm{Co}_{2} \mathrm{MnSi}$ and $\mathrm{Co}_{2} \mathrm{FeSi}$, accounting for the $40-60 \%$ mixture of $\mathrm{Fe}$ and $\mathrm{Mn}$.

The first row of Fig. 6 shows the as-calculated bands, the second row the same bands adjusted with the measured band positions at $\Gamma$. Each of the band groups $A-C, D-E, F-H, I-J$, and $K-M$ is degenerate at the $\Gamma$ point. In Figs. 6(b) and $6(\mathrm{~d})$ all majority bands coincide in a small energy interval at $\Gamma$. As all band groups have been identified in the experiment, we can adjust their binding energies to the measured band positions close to the $\Gamma$ point [Figs. $6(\mathrm{~g})-6(1)$ and Table I]. Note that we have assumed probing the $\Gamma K X$ plane for all three compounds.

For $\mathrm{Co}_{2} \mathrm{MnGa}$, the shifts for the frontier bands $A-C$ and $D-E$ near $\Gamma$ close to $E_{\mathrm{F}}$ go in opposite directions, i.e., for small momentum values the deficiency in filling of the majority bands is compensated by excess charge in the top minority bands. Majority bands $I-J$ and minority bands $K-M$ have reversed their order in the adjusted bands. The top of the minority band lies only $0.2 \mathrm{eV}$ above $E_{\mathrm{F}}$ (instead of $0.45 \mathrm{eV}$ in the calculation), i.e., this material is much closer to being half-metallic ferromagnetic than predicted. This explains why the measured magnetic moment of $4.0 \pm 0.1 \mu_{\mathrm{B}}$ [78] is so close to the prediction of the Slater-Pauling rule, which would not be valid if the minority bands are significantly unoccupied. The experimental exchange splitting of the frontier bands $A-C$ and $F-H$ at the $\Gamma$ point $\left[\Delta E_{\mathrm{ex}}=0.48 \pm 0.07 \mathrm{eV}\right.$; see dotted lines in Figs. 6(g) and 6(h)] is almost a factor of 2 smaller than 
the predicted splitting of $0.85 \mathrm{eV}$ in the as-calculated bands [Figs. 6(a) and 6(b)].

For $\mathrm{Co}_{2} \mathrm{MnSi}$ the experimental exchange splitting $\left[\Delta E_{\mathrm{ex}}=\right.$ $0.5 \pm 0.1 \mathrm{eV}$; see dotted lines in Figs. 6(i) and 6(j)] of frontier bands $A-C$ and $F-H$ at $\Gamma$ is also significantly smaller than the value of $0.93 \mathrm{eV}$ predicted by the DFT-LSDA calculation. The measured minority spin gap $(0.50 \mathrm{eV})$ is twice as large as the calculated one $\left(0.25 \mathrm{eV}\right.$; both referenced to $\left.E_{\mathrm{F}}\right)$. For $\mathrm{Co}_{2} \mathrm{MnSi}$ a number of calculations with different approximations exist. We will compare the results of ten different calculations with experiment in the next section.

For $\mathrm{Co}_{2} \mathrm{Fe}_{0.4} \mathrm{Mn}_{0.6} \mathrm{Si}$ the experimental exchange splitting $\left[\Delta E_{\mathrm{ex}}=0.75 \pm 0.05 \mathrm{eV}\right.$; see dotted lines in Figs. $6(\mathrm{k})$ and 6(1)] between frontier bands $A-C$ and $F-H$ at $\Gamma$ is again smaller than predicted $\left(1.02 \mathrm{eV}\right.$ for $\left.\mathrm{Co}_{2} \mathrm{FeSi}\right)$. The upward shift of the majority bands near $\Gamma$ has increased further in comparison with $\mathrm{Co}_{2} \mathrm{MnGa}$ and $\mathrm{Co}_{2} \mathrm{MnSi}$. In contrast to $\mathrm{Co}_{2} \mathrm{MnGa}$ and $\mathrm{Co}_{2} \mathrm{MnSi}$, minority bands $A-C$ for $\mathrm{Co}_{2} \mathrm{Fe}_{0.4} \mathrm{Mn}_{0.6} \mathrm{Si}$ are shifted upwards, too. The measured minority spin gap $(0.35 \mathrm{eV})$ is almost a factor of 2 smaller than the calculated one $(0.7 \mathrm{eV}$; referenced to the interpolated $E_{\mathrm{F}}$ ).

The differences in the positions of the experimental and theoretical Fermi levels in Fig. 6 have important implications. The minority spin gap is relevant for macroscopic properties, among all for the transport properties. The many-body electron correlations influence the size of the minority gap. These correlations are subject to simplifying assumptions in theoretical approaches. Our results indicate that current theoretical models qualitatively explain the experimental observations, however, there is still a lack of quantitative agreement. It is remarkable that the magnetic moments are predicted in good agreement with the experimental values, a fact that occurs already for common DFT-LSDA calculations using standard exchange correlation potentials. This can be explained as follows: For compounds exhibiting a minority gap at the Fermi level, i.e., half-metallic ferromagnets, the magnetic moment directly results from the Slater-Pauling rule $[1,76]$. For compounds, which are not half-metallic, the explanation for the good agreement for the magnetic moments is not so straightforward and in fact the agreement is not as good, e.g., for $\mathrm{Co}_{2} \mathrm{CrAl}$ [79]. However, as long as the minority bands are almost filled (or are just filled a little), the influence on the total magnetic moment is quite small. For $\mathrm{Co}_{2} \mathrm{MnGa}$ the measured magnetic moment is $4.0 \mu_{\mathrm{B}}$ per formula unit [78], in agreement with the theoretical prediction of $4.1 \mu_{\mathrm{B}}$ [80]. This is in accordance with our finding that only a little part of the minority band is unoccupied.

The comparison of experiment and DFT-LSDA calculation revealed that the shifts for majority and minority bands are different (in some cases even in sign) and different band groups require different shifts. This finding deviates from the anticipated behavior on the basis of a rigid-band description. Moreover the correlations not only shift the bands but also change their dispersion.

\section{B. Band structure calculations for $\mathrm{Co}_{2} \mathrm{MnSi}$ with various approximations}

$\mathrm{Co}_{2} \mathrm{MnSi}$ is the half-metallic ferromagnetic Heusler compound most investigated by theory. Several approximations
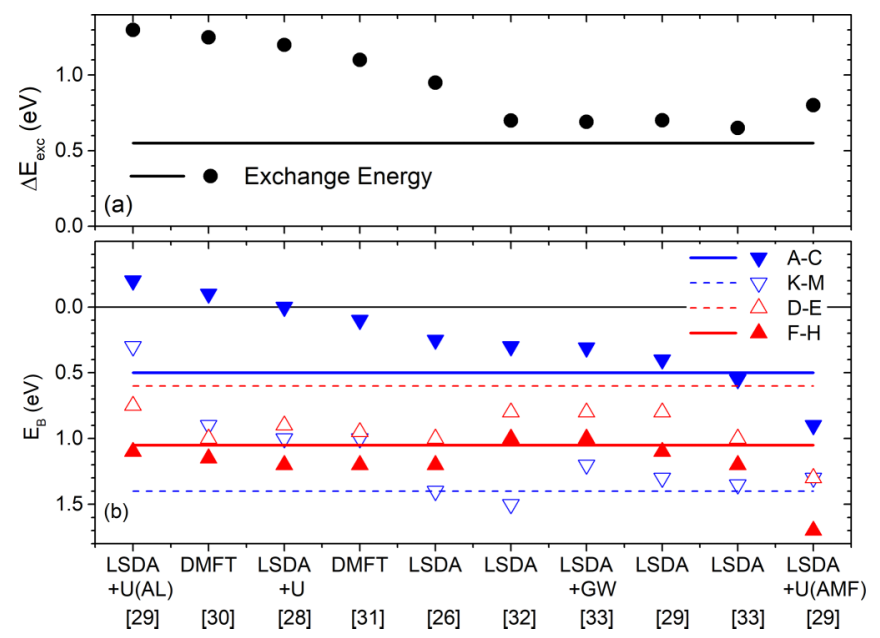

FIG. 7. Comparison of experimental exchange splitting (a) and binding energies of band groups (b) with computational results from ten different calculations. Full and dashed lines represent the experimental results (blue for minority and red for majority bands); dots (in a) and colored triangles (in b) are taken from calculations. The values for minority bands $A-C$ and $K-M$ and majority bands $D-E$ and $F-H$ refer to binding energies at the $\Gamma$ point. The band edge of the topmost occupied minority band $A-C$ (full blue triangles, full blue line) determines the onset of the minority spin gap. The exchange splitting in (a) is determined from the difference of minority band edge $A-C$ and corresponding majority band edge $F-H$.

have been employed with the aim to capture the many-body correlation effects. The approximations turned out to be challenging because the valence band states are partly itinerant and partly localized. The present results provide an excellent data set for a quantitative comparison with calculations using different codes for the inclusion of electron correlations. Data for Refs. [28,29,33] are estimated from the density of states (DOS) function; all other references provided a direct graphical representation of band dispersions. The unambiguous extraction of band positions from the DOS is in general not possible. In this case, we can estimate the band position because despite variations of bandwidth and energy shifts the band structure of various calculations appears similar to each other.

Figure 7 compares the theoretical results from ten different calculations with the present experimental data. Figure 7(a) shows the values for the exchange splitting $\Delta E_{\text {ex }}$ in comparison with experiment (full line). It is evident that all calculations overestimate the size of the exchange splitting, with some of them coming close to the experimental value of $0.55 \mathrm{eV}$. Figure 7(b) shows the binding energies of the maxima of minority band groups $A-C$ and $K-M$ (blue) and the energies of majority band groups $D-E$ and $F-H$ (red) at the $\Gamma$ point. In order to elucidate a possible systematic trend, the calculated results are sorted by increasing binding energy of the topmost minority bands $A-C$ (full blue triangles). The predicted positions of bands $A-C$ cover a wide range from $0.2 \mathrm{eV}$ above $E_{\mathrm{F}}$ (left side) to $0.9 \mathrm{eV}$ below $E_{\mathrm{F}}$ (right side). The maximum of $A-C$ represents the highest energy level occupied by minority electrons, i.e., it defines the minority spin gap. Positions below $E_{\mathrm{B}}=0$ indicate half-metallic ferromagnetic 
behavior. The measured position of the top of $A-C$ is $0.5 \mathrm{eV}$ (full blue line), meaning that most calculations underestimate the spin gap (with respect to $E_{\mathrm{F}}$ ). The three calculations on the left-hand side do not predict a spin gap at all.

We briefly recall the essential differences of the codes used for the calculations. Reference [29] uses a combination of first-principles DFT with Hubbard models, which are widely used to investigate the properties of strongly correlated materials. This calculation is based on a self-consistent local density approximation (LSDA) and considers additionally the Hubbard $U$ in the atomic limit (AL) and around-mean-field (AMF) functionals for the double counting term. This term is used to subtract the correlation part from the total energy. The results are compared to the conventional LSDA calculations. In Ref. [30] the electronic structure calculations were performed within the full-potential spin-polarized relativistic Korringa-Kohn-Rostoker (SPR-KKR) method. Local correlations were considered within the dynamical mean field theory (DMFT) scheme implemented within the KKR method. For the case of $\mathrm{Co}_{2} \mathrm{FeSi}$, it has been found that the local spin density approximation (LSDA) is not sufficient for describing the electronic structure correctly [28], but significant improvement was suggested for adding an orbital-dependent electron-electron correlation (LSDA $+U$ ). A similar LSDA + DMFT approach to that in Ref. [30] has been used in Ref. [31]. In this case the exchange and correlation potential in the Vosko, Wilk, and Nusair parametrization has been used, in contrast to the usual Perdew-Burke-Ernzerhof (PBE) parametrization. The self-consistent calculations based on LSDA [26] have already been discussed above. Reference [32] performed ab initio calculations, using LSDA, and employs the von Barth-Hedin parametrization for the exchange correlation potential. Reference [33] compares LSDA- PBE parametrization with quasiparticle spectra calculated within the one-shot Green's function and screened Coulomb interaction (GW) approximation. The GW approximation yields the quasiparticle energies as corrections to the Kohn-Sham energies and represents an alternative parameter-free approach to local correlations. In Refs. [26,28,29,33] spin-orbit interaction is not considered, while it is included in the calculations reported in Refs. [30-32].

The theoretical results reported in Refs. [29,32,33] show the best agreement with the experiment. Local correlations $(+U)$ as well as DMFT show larger deviations and overestimate the exchange energy. Part of the theoretical results even do not find half-metallic ferromagnetism for $\mathrm{Co}_{2} \mathrm{MnSi}$. The smallest mean deviation for the four bands considered here is $0.2 \mathrm{eV}$, which is larger than the experimental uncertainty of $0.1 \mathrm{eV}$. Thus, none of the theoretical results exactly reproduces the experimental data. Note that all theoretical results predict the experimental magnetization according to the Slater-Pauling rule of $5 \mu_{\mathrm{B}}$ per formula unit. Moreover, several theoretical results $[28,30,31,33]$ are compared to experimental results, such as momentum-integrated or normal emission photoelectron spectroscopy data, and claim good agreement. We note that these averaged properties are not sensitive enough to capture variations due to different approximations for considering electron-electron correlation effects.

\section{Role of quasielastic spin-conserving phonon scattering}

The entire data paraboloids exhibit a majority-spin density as diffuse background underlying the band features. This is most clearly visible for the two half-metallic ferromagnets in the constant-energy cuts between $E_{\mathrm{F}}$ and the top of the minority bands [see Figs. 3(q)-3(w) and videos in the Supplemental Material [75]]. The steep downward-dispersing theoretical majority bands close to the $K$ and $X$ points [Figs. $6(\mathrm{~b})$, 6(d), and 6(f)] lie outside the photoemission horizon and thus these bands are not directly visible in the $k$ field of $<0.7 \AA^{-1}$ radius. Electrons from these bands are excited at $h v=6.05 \mathrm{eV}$ but their momentum component perpendicular to the surface is too small, so they cannot escape through the surface barrier. Nevertheless, these bands can be the origin of the experimentally observed majority background due to $k$-randomizing scattering processes. Quasielastic phonon or defect scattering can transfer large momenta but the energy transfer is very small. This indirect photoemission channel has been studied and discussed in detail at higher energies, where it can even dominate over the direct photoemission channel. The resulting background carries the signature of the matrix-element-weighted density of states (MEWDOS) [81-83].

Spin-conserving processes of this type can scatter the electrons towards all directions and thus cause a homogeneous background, which carries the spin character of the majority bands at the same energy outside the photoemission horizon. At low energies like $6.05 \mathrm{eV}$ the phonon-scattering cross section is small. Hence this highly polarized background is weak in comparison with the band intensities [Figs. 3(f), 3(g), 3(j), 3(k), 3(r), 3(s), 3(v), and 3(w)]. For $\mathrm{Co}_{2} \mathrm{Fe}_{0.4} \mathrm{Mn}_{0.6} \mathrm{Si}$ this majority-spin background is significant in the entire spectrum because the disorder by the random distribution of $\mathrm{Fe}$ and $\mathrm{Mn}$ on identical lattice sites gives rise to an additional defect-scattering probability in comparison with $\mathrm{Co}_{2} \mathrm{MnSi}$. Most likely, quasielastic spin-conserving phononor defect-scattering dominates over the competing mechanism of spin-selective processes leading to the transport spin polarization as discussed in Ref. [84].

From the view of quantum mechanics, such stochastic events destroy the coherence of the wave packet of the scattered electron with the (probability) wave of the total final state. This, in turn, extinguishes the electron's memory on its initial momentum vector; the momentum is transferred to the phonon bath. Not much is known about such processes, but it is evident that the energy transfer is small (quasielastic) and the spin orientation might be retained if the spin-flip amplitudes are small.

The large IMFP at these very low energies not only emphasizes bulk states at the expense of surface states but it also increases the probability for phonon scattering, a wellknown fact in high-energy photoemission. A fingerprint of such $k$-randomizing phonon-scattering processes is the occurrence of a substantial magnetic circular dichroism (MCD) in photoemission at very low energies $[85,86]$. Both the spin polarization and the MCD strongly suggest that such scattering processes are significant in the near-threshold region. 


\section{SUMMARY AND CONCLUSIONS}

Spin- and momentum-resolved photoemission for in situ deposited epitaxial films of $\mathrm{Co}_{2} \mathrm{MnGa}, \mathrm{Co}_{2} \mathrm{MnSi}$, and $\mathrm{Co}_{2} \mathrm{Fe}_{0.4} \mathrm{Mn}_{0.6} \mathrm{Si}$ on $\mathrm{MgO}(100)$ revealed the bulk electronic structure including the spin character of the topmost valence bands. Following the Slater-Pauling rule, these full-Heusler compounds have valence electron numbers of 4, 5, and 5.4, representing the numbers of Bohr magnetons per formula unit [24,87]. The central aim was to track the tuning of the electronic bands with increasing band filling.

Systematic measurements with different photon polarizations exploit symmetry selection rules to analyze closelyspaced bands with different spin character. Complementary spin-integrated hard x-ray momentum microscopy reveals band dispersions in a larger momentum range.

The results for $\mathrm{Co}_{2} \mathrm{MnGa}$ reveal a one-to-one agreement (including spin signature) of the experimental band patterns with the bands predicted by the DFT-LSDA calculation [26], after adjustment of the energetic band positions. This agreement served as basis for a band assignment, which is adopted for the two other materials. Additional (spin-integral) measurements with the same technique using hard $\mathrm{x}$ rays (beamline P22 at PETRA III, Hamburg), where the photon energy has been tuned to probe the $\Gamma-K-X$ plane, show a good agreement with results obtained with the phototransition at $6.05 \mathrm{eV}$. This agreement indicates that with 6.05 $\mathrm{eV}$ one approximately probes the plane containing the $\Gamma-K$ and $\Gamma-X$ directions in $k$ space. For $\mathrm{Co}_{2} \mathrm{MnGa}$, the topmost occupied minority band, which is most important for the transport properties and possible applications, crosses $E_{\mathrm{F}}$ with a parallel momentum of $0.22 \AA^{-1}$ along $\Gamma-K$, thus excluding half-metallic ferromagnetic behavior. This result is in good agreement with the finding of Ref. [58]. Pinpointing the topmost occupied minority band for the two other compounds well below $E_{\mathrm{F}}$ proves that both are half-metallic ferromagnets with minority spin gaps (with respect to $E_{\mathrm{F}}$ ) of 0.5 and $0.35 \mathrm{eV}$ for $\mathrm{Co}_{2} \mathrm{MnSi}$ and $\mathrm{Co}_{2} \mathrm{Fe}_{0.4} \mathrm{Mn}_{0.6} \mathrm{Si}$, respectively. In the framework of a simple band-filling picture, one would expect the opposite trend, reflecting the increasing number of valence electrons. The measured exchange splitting energies of $0.48,0.55$, and $0.75 \mathrm{eV}$ for $\mathrm{Co}_{2} \mathrm{MnGa}, \mathrm{Co}_{2} \mathrm{MnSi}$, and $\mathrm{Co}_{2} \mathrm{Fe}_{0.4} \mathrm{Mn}_{0.6} \mathrm{Si}$, respectively, are significantly smaller than the values predicted by the DFT-LSDA calculation $(0.85$, 0.93 , and $1.02 \mathrm{eV}$ ).

When comparing results of different experiments, it is crucial to consider the region in $k$ space which is probed at a given photon energy. A transition scheme in 3D momentum space [72] with the parameters of the title compounds uncovers that the present low-energy and hard x-ray experiments and that of Refs. [54,55] probes the region close to the $\Gamma-X-K$ plane, i.e., the center plane of the bulk BZ. The experiments of Refs. [21,34] in the VUV range probe regions close to the $X-W-U$ plane [top and bottom squares in
Fig. 2(o)]. Photon energies of $h v=21.2$ and $37.0 \mathrm{eV}$ result in $k_{z}=2.91 \AA^{-1}=1.34 G_{100}$ and $3.57 \AA^{-1}=1.64 G_{100}$, respectively. We conclude that these four experiments probe different sections of $k$ space, so we cannot expect agreement of the measured spectra. For more details about this description of direct transitions in $k$ microscopy, see Ref. [72].

Both half-metallic ferromagnetic compounds exhibit a significant diffuse majority-spin background throughout the entire $\left(E_{\mathrm{B}}, \boldsymbol{k}\right)$ parameter space of the recorded data arrays. We attribute this background to $k$ randomizing but spinconserving stochastic scattering processes of excited majority electrons from bands outside of the visible photoemission horizon. The spectral distribution reflects the matrix-elementweighted DOS as known from experiments at high energies [81-83]. The diffuse majority-spin density is much stronger for $\mathrm{Co}_{2} \mathrm{Fe}_{0.4} \mathrm{Mn}_{0.6} \mathrm{Si}$, probably because stochastic scattering is enhanced due to the chemical disorder of the $\mathrm{Fe}$ and Mn distribution. It is likely that this kind of scattering process also affects spin-dependent transport processes in such half-metallic ferromagnets. This issue could be addressed by femtosecond spin dynamics in the time domain with high $k$ resolution. Such experiments would be highly desirable to elucidate the link between electronic structure, spin transport, and ultralow magnetic damping [21] and are just becoming feasible at free-electron laser sources [65].

The central result of this work is that the electronic bands in Heusler compounds indeed systematically vary with the number of valence electrons. However, the variation does not follow a rigid common shift for all bands as suggested by the rigid-band model. Instead, the increasing exchange splitting with increasing magnetic moment overcompensates for minority bands the trend of decreasing energy with increasing number of valence electrons. The comparison of theoretical predictions calculated by various density functional models with our experimental results reveals sizable discrepancies of exchange energies, minority spin gaps, and band dispersions. Our results confirm that tuning of the band structure of metallic compounds by partial replacement of specific elements is experimentally feasible. However, we show that the obtained modifications of the electronic properties are far more complex than simple rigid-band models predict and still pose a challenge for band structure calculations.

\section{ACKNOWLEDGMENTS}

We thank A. Oelsner, Surface Concept GmbH, Mainz for continuous support of the experiments. Further thanks go to C. Schlueter, A. Gloskovskii, and Yu. Matveyev for excellent support during the HAXPES experiment (Figs. 4 and 5) at beamline P22 of PETRA III (DESY, Hamburg). Financial support by Deutsche Forschungsgemeinschaft (DFG, German Research Foundation) - TRR 173 Spin+X - 268565370 (Projects No. A02, No. A05, and No. A09) as well as BMBF (Projects No. 05K16UMC and No. 05K19UM2) is gratefully acknowledged.
[1] T. Graf, C. Felser, and S. S. P. Parkin, Simple rules for the understanding of heusler compounds, Prog. Solid State Chem. 39, 1 (2011).
[2] L. Wollmann, A. K. Nayak, S. S. P. Parkin, and C. Felser, Heusler 4.0: tunable materials, Annu. Rev. Mater. Res. 47, 247 (2017). 
[3] T. Graf, S. S. P. Parkin, and C. Felser, Heusler compounds-a material class with exceptional properties, IEEE Trans. Magn. 47, 367 (2011).

[4] K. Manna, Y. Sun, L. Müchler, J. Kübler, C. Felser, Heusler, Weyl, and Berry, Nat. Rev. Mater. 3, 244 (2018).

[5] A. Heusler, Über magnetische manganlegierungen, Verh. Dtsch. Phys. Ges. 5, 219 (1903).

[6] C. Shekhar, M. Nicklas, A. K. Nayak, S. Ouardi, W. Schnelle, G. Fecher, C. Felser, and K. Kobayashi, Electronic structure and nonsaturating magnetoresistance of superconducting heusler topological insulators. J. Appl. Phys. 113, 17E142 (2013).

[7] Y. Nakajima, R. Hu, K. Kirshenbaum, A. Hughes, P. Syers, X. Wang, K. Wang, R. Wang, S. R. Saha, D. Pratt, J. W. Lynn, and J. Paglione, Topological RPdBi half-heusler semimetals: a new family of noncentrosymmetric magnetic superconductors, Sci. Adv. 1, e1500242 (2015).

[8] Z. K. Liu, L. X. Yang, S.-C. Wu, C. Shekhar, J. Jiang, H. F. Yang, Y. Zhang, S.-K. Mo, Z. Hussain, B. Yan, C. Felser, and Y. L. Chen, Observation of unusual topological surface states in half-Heusler compounds LnPtBi ( $\mathrm{Ln}=\mathrm{Lu}, \mathrm{Y})$, Nat. Commun. 7, 12924 (2016).

[9] B. Yan and A. de Visser, Half-heusler topological insulators, MRS Bull. 39, 859 (2014).

[10] Y. Pan, A. M. Nikitin, T. V. Bay, Y. K. Huang, C. Paulsen, B. H. Yan, and A. de Visser, Superconductivity and magnetic order in the noncentrosymmetric half-heusler compound ErPdBi, Europhys. Lett. 104, 27001 (2013).

[11] A. Kronenberg, J. Braun, J. Minar, H.-J. Elmers, D. Kutnyakhov, A. V. Zaporozhchenko, R. Wallauer, S. Chernov, K. Medjanik, G. Schönhense, M. Kläui, S. Chadov, H. Ebert, and M. Jourdan, Dirac cone and pseudo-gapped density of states in the topological half-Heusler compound YPtBi, Phys. Rev. B 94, 161108(R) (2016).

[12] I. Žutić, J. Fabian, and S. Das Sarma, Spintronics: fundamentals and applications, Rev. Mod. Phys. 76, 323 (2004).

[13] S. A. Wolf, D. D. Awschalom, R. A. Buhrman, J. M. Daughton, S. von Molnár, M. L. Roukes, A. Y. Chtchelkanova, and D. M. Treger, Spintronics: a spin-based electronics vision for the future, Science 294, 1488 (2001).

[14] G. A. Prinz, Magnetoelectron. Sci. 282, 1660 (1998).

[15] Y. Sakuraba, M. Hattori, M. Oogane, Y. Ando, H. Kato, A. Sakuma, T. Miyazaki, and H. Kubota, Giant tunneling magnetoresistance in $\mathrm{Co}_{2} \mathrm{MnSi} / \mathrm{Al}-\mathrm{O} / \mathrm{Co}_{2} \mathrm{MnSi}$ magnetic tunnel junctions, Appl. Phys. Lett. 88, 192508 (2006).

[16] H. Liu, Y. Honda, T. Taira, K. Matsuda, M. Arita, T. Uemura, and $\mathrm{M}$. Yamamoto, Giant tunneling magnetoresistance in epitaxial $\mathrm{Co}_{2} \mathrm{MnSi} / \mathrm{MgO} / \mathrm{Co}_{2} \mathrm{MnSi}$ magnetic tunnel junctions by half-metallicity of $\mathrm{Co}_{2} \mathrm{MnSi}$ and coherent tunneling, Appl. Phys. Lett. 101, 132418 (2012).

[17] Y. Sakuraba, K. Izumi, T. Iwase, S. Bosu, K. Saito,K. Takanashi, Y. Miura, K. Futatsukawa, K. Abe, and M. Shirai, Mechanism of large magnetoresistance in $\mathrm{Co}_{2} \mathrm{MnSi} / \mathrm{Ag} / \mathrm{Co}_{2} \mathrm{MnSi}$ devices with current perpendicular to the plane, Phys. Rev. B 82, 094444 (2010).

[18] J. Sato, M. Oogane, H. Naganuma, and Y. Ando, Large magnetoresistance effect in epitaxial $\mathrm{Co}_{2} \mathrm{Fe}_{0.4} \mathrm{Mn}_{0.6} \mathrm{Si} / \mathrm{Ag} / \mathrm{Co}_{2} \mathrm{Fe}_{0.4} \mathrm{Mn}_{0.6} \mathrm{Si}$ Devices, Appl. Phys. Express 4, 113005 (2011).

[19] M. J. Carey, S. Maat, S. Chandrashekariaih, J. A. Katine, W. Chen, B. York, and J. R. Childress, $\mathrm{Co}_{2} \mathrm{MnGe}$-based current- perpendicular-to-the-plane giant-magnetoresistance spin-valve sensors for recording head applications, J. Appl. Phys. 109, 093912 (2011).

[20] R. A. de Groot, F. M. Mueller, P. G. van Engen, and K. H. J. Buschow, New Class of Materials: Half-Metallic Ferromagnets, Phys. Rev. Lett. 50, 2024 (1983).

[21] C. Guillemard, S. Petit-Watelot, L. Pasquier, D. Pierre, J. Ghanbaja, J-C. Rojas-Sánchez, A. Bataille, J. Rault, P. Le Fèvre, F. Bertran, and S. Andrieu, Ultralow Magnetic Damping in $\mathrm{Co}_{2} \mathrm{Mn}$-Based Heusler Compounds: Promising Materials for Spintronics, Phys. Rev. Appl. 11, 064009 (2019).

[22] B. Balke, S. Wurmehl, G. H. Fecher, C. Felser, and J. Kübler, Rational design of new materials for spintronics: $\mathrm{Co}_{2} \mathrm{Fe} Z$ $(Z=\mathrm{Al}, \mathrm{Ga}, \mathrm{Si}, \mathrm{Ge})^{*}$, Sci. Technol. Adv. Mater. 9, 014102 (2008).

[23] S. Fujii, S. Sugimura, Ishida, and S. Asano, Hyperfine fields and electronic structures of the Heusler alloys $\mathrm{Co}_{2} \mathrm{MnX}(X=\mathrm{Al}$, Ga, Si, Ge, Sn), J. Phys.: Condens. Matter 2, 8583 (1990).

[24] I. Galanakis, P. H. Dederichs, and N. Papanikolaou, SlaterPauling behavior and origin of the half-metallicity of the full-heusler alloys, Phys. Rev. B 66, 174429 (2002).

[25] C. Lidig, J. Minár, J. Braun, H. Ebert, A. Gloskovskii, A. Kronenberg, M. Kläui, and M. Jourdan. Signature of a highly spin polarized resonance state at $\mathrm{Co}_{2} \mathrm{MnSi}(001) / \mathrm{Ag}(001)$ interfaces, J. Phys. D: Appl. Phys. 51, 135307 (2018).

[26] J. Ma, V. I. Hegde, K. Munira, Y. Xie, S. Keshavarz, D. T. Mildebrath, C. Wolverton, A. W. Ghosh, and W. H. Butler, Computational investigation of half-heusler compounds for spintronics applications, Phys. Rev. B 95, 024411 (2017); http: //heusleralloys.mint.ua.edu/.

[27] I. Galanakis, P. Mavropoulos, and P. H. Dederichs, Electronic structure and slater-pauling behaviour in half-metallic Heusler alloys calculated from first principles, J. Phys. D: Appl. Phys. 39, 765 (2006).

[28] B. Balke, G. H. Fecher, H. C. Kandpal, C. Felser, K. Kobayashi, E. Ikenaga, J. J. Kim, and S. Ueda, Properties of the quaternary half-metal-type Heusler alloy $\mathrm{Co}_{2} \mathrm{Mn}_{1-x} \mathrm{Fe}_{x} \mathrm{~S}$, Phys. Rev. B 74, 104405 (2006).

[29] C. Tsirogiannis and I. Galanakis, Effect of the double-counting functional on the electronic and magnetic properties of halfmetallic magnets using the GGA+U method, J. Magn. Magn. Mater. 393, 297 (2015).

[30] H. Schneider, E. Vilanova-Vidal, S. Chadov, G. H. Fecher, C. Felser, and G. Jakob, Hall effect and electronic structure of $\mathrm{Co}_{2} \mathrm{Fe}_{x} \mathrm{Mn}_{1-x} \mathrm{Si}$ films, J. Magn. Magn. Mater. 322, 579 (2010).

[31] J.-P. Wüstenberg, R. Fetzer, M. Aeschlimann, M. Cinchetti, J. Minar, J. Braun, H. Ebert, T. Ishikawa, T. Uemura, and M. Yamamoto, Surface spin polarization of the nonstoichiometric Heusler alloy $\mathrm{Co}_{2} \mathrm{MnSi}$, Phys. Rev. B 85, 064407 (2012).

[32] S. Picozzi, A. Continenza, and A. J. Freeman, Magneto-optical properties of heusler compounds from a first-principles approach, J. Phys. D: Appl. Phys. 39, 851 (2006).

[33] M. Meinert, C. Friedrich, G. Reiss, and S. Blügel, GW study of the half-metallic heusler compounds $\mathrm{Co}_{2} \mathrm{MnSi}$ and $\mathrm{Co}_{2} \mathrm{FeSi}$, Phys. Rev. B 86, 245115 (2012).

[34] M. Jourdan, J. Minár, J. Braun, A. Kronenberg, S. Chadov, B. Balke, A. Gloskovskii, M. Kolbe, H. J. Elmers, G. Schönhense, H. Ebert, C. Felser, and M. Kläui, Direct observation of halfmetallicity in the heusler compound $\mathrm{Co}_{2} \mathrm{MnSi}$, Nat. Commun. 5, 3974 (2014). 
[35] S. Andrieu, A. Neggache, T. Hauet, T. Devolder, A. Hallal, M. Chshiev, A. M. Bataille, P. Le Fèvre, and F. Bertran, Direct evidence for minority spin gap in the $\mathrm{Co}_{2} \mathrm{MnSi}$ heusler compound, Phys. Rev. B 93, 094417 (2016).

[36] T. Akiho, J. Shan, H. X. Liu, K. I. Matsuda, M. Yamamoto, and T. Uemura, Electrical injection of spin-polarized electrons and electrical detection of dynamic nuclear polarization using a Heusler alloy spin source, Phys. Rev. B 87, 235205 (2013).

[37] S. Picozzi, A. Continenza, and A. J. Freeman, $\mathrm{Co}_{2} \mathrm{Mn} X(X=$ $\mathrm{Si}, \mathrm{Ge}, \mathrm{Sn}$ ) heusler compounds: An ab initio study of their structural, electronic, and magnetic properties at zero and elevated pressure, Phys. Rev. B 66, 094421 (2002).

[38] S. Ishida, S. Fujii, S. Kashiwagi, and S. Asano, Search for halfmetallic compounds in $\mathrm{Co}_{2} \mathrm{MnZ}(Z=\mathrm{IIIb}, \mathrm{IVb}, \mathrm{Vb}$ Element), J. Phys. Soc. Jpn. 64, 2152 (1995).

[39] P. J. Brown, K. U. Neumann, P. J. Webster, and K. R. A. Ziebeck, The magnetization distributions in some Heusler alloys proposed as half-metallic ferromagnets, J. Phys.: Condens. Matter 12, 1827 (2000).

[40] L. Ritchie, G. Xiao, Y. Ji, T. Y. Chen, C. L. Chien, M. Zhang, J. Chen, Z. Liu, G. Wu, and X. X. Zhang, Magnetic, structural, and transport properties of the Heusler alloys $\mathrm{Co}_{2} \mathrm{MnSi}$ and NiMnSb, Phys. Rev. B 68, 104430 (2003).

[41] Y. Sakuraba, J. Nakata, M. Oogane, H. Kubota, Y. Ando, A. Sakuma, and T. Miyazaki, Huge spin-polarization of L21Ordered $\mathrm{Co}_{2} \mathrm{MnSi}$ epitaxial heusler alloy film, Jpn. J. Appl. Phys. 44, L1100 (2005).

[42] L. J. Singh, Z. H. Barber, Y. Miyoshi, Y. Bugoslavsky, W. R. Branford, and L. F. Cohen, Structural, magnetic, and transport properties of thin films of the heusler alloy $\mathrm{Co}_{2} \mathrm{MnSi}$, Appl. Phys. Lett. 84, 2367 (2004).

[43] S. Kämmerer, A. Thomas, A. Hütten, and G. Reiss, $\mathrm{Co}_{2} \mathrm{MnSi}$ heusler alloy as magnetic electrodes in magnetic tunnel junctions, Appl. Phys. Lett. 85, 79 (2004).

[44] W. H. Wang, M. Przybylski, W. Kuch, L. I. Chelaru, J. Wang, Y. F. Lu, J. Barthel, H. L. Meyerheim, and J. Kirschner, Magnetic properties and spin polarization of $\mathrm{Co}_{2} \mathrm{MnSi}$ Heusler alloy thin films epitaxially grown on GaAs(001), Phys. Rev. B 71, 144416 (2005)

[45] N. J. Harmon, T. A. Peterson, C. C. Geppert, S. J. Patel, C. J. Palmstrøm, P. A. Crowell, and M. E. Flatte, Anisotropic spin relaxation in $\mathrm{n}$-GaAs from strong inhomogeneous hyperfine fields produced by the dynamical polarization of nuclei, Phys. Rev. B 92, 140201(R) (2015).

[46] C. Liu, S. J. Patel, T. A. Peterson, C. C. Geppert, K. D. Christie, G. Stecklein, C. J. Palmstrøm, and P. A. Crowell, Dynamic detection of electron spin accumulation in ferromagnet-semiconductor devices by ferromagnetic resonance, Nat. Commun. 7, 10296 (2016).

[47] G. Chang, S.-Y. Xu, X. Zhou, S.-M. Huang, B. Singh, B. Wang, I. Belopolski, J. Yin, S. Zhang, A. Bansil, H. Lin, and M. Z. Hasan, Topological Hopf and Chain Link Semimetal States and Their Application to $\mathrm{Co}_{2} \mathrm{MnGa}$, Phys. Rev. Lett. 119, 156401 (2017).

[48] I. Belopolski, D. S. Sanchez, G. Chang, K. Manna, B. Ernst, S.-Y. Xu, S. S. Zhang, H. Zheng, J. Yin, B. Singh, G. Bian, D. Multer, X. Zhou, S.-M. Huang, B. Wang, A. Bansil, H. Lin, C. Felser, and M. Z. Hasan, A three-dimensional magnetic topological phase, Science 365, 1278 (2019).
[49] J. Braun, M. Jourdan, A. Kronenberg, S. Chadov, B. Balke, M. Kolbe, A. Gloskovskii, H. J. Elmers, G. Schönhense, C. Felser, M. Kläui, H. Ebert, and J. Minár, Monitoring surface resonances on $\mathrm{Co}_{2} \mathrm{MnSi}(100)$ by spin-resolved photoelectron spectroscopy, Phys. Rev. B 91, 195128 (2015).

[50] C. Lidig, J. Minár, J. Braun, H. Ebert, A. Gloskovskii, J. A. Krieger, V. Strocov, M. Kläui, and M. Jourdan, Surface resonance of thin films of the heusler half-metal $\mathrm{Co}_{2} \mathrm{MnSi}$ probed by soft x-ray angular resolved photoemission spectroscopy, Phys. Rev. B 99, 174432 (2019).

[51] M. Kolbe, S. Chadov, E. A. Jorge, G. Schönhense, C. Felser, H.-J. Elmers, M. Kläui, and M. Jourdan, Test of band structure calculations for heusler compounds by spin-resolved photoemission spectroscopy, Phys. Rev. B 86, 024422 (2012).

[52] C. Guillemard, S. Petit-Watelot, J.-C. Rojas-Sánchez, J. Hohlfeld, J. Ghanbaja, A. Bataille, P. Le Fèvre, F. Bertran, and $\mathrm{S}$. Andrieu, Polycrystalline $\mathrm{Co}_{2} \mathrm{Mn}$-based heusler thin films with high spin polarization and low magnetic damping, Appl. Phys. Lett. 115, 172401 (2019).

[53] S. Tanuma et al., Calculations of electron inelastic mean free paths. IX. Data for 41 elemental solids over the $50 \mathrm{eV}$ to $30 \mathrm{keV}$ range, Surf. Interface Anal. 43, 689 (2011); C. J. Powell and S. Tanuma, in Hard X-ray Photoelectron Spectroscopy (HAXPES), Springer Series in Surface Sciences Vol. 59, edited by J. C. Woicik (Springer, Cham, Switzerland, 2016), Chap. 4.

[54] R. Fetzer, J.-P. Wüstenberg, T. Taira, T. Uemura, M. Yamamoto, M. Aeschlimann, and M. Cinchetti, Structural, chemical, and electronic properties of the $\mathrm{Co}_{2} \mathrm{MnSi}(001) / \mathrm{MgO}$ interface. Phys. Rev. B 87, 184418 (2013).

[55] R. Fetzer, B. Stadtmüller, Y. Ohdaira, H. Naganuma, M. Oogane, Y. Ando, T. Taira, T. Uemura, M. Yamamoto, M. Aeschlimann, and M. Cinchetti, Probing the electronic and spintronic properties of buried interfaces by extremely low energy photoemission spectroscopy, Sci. Rep. 5, 8537 (2015).

[56] K. Miyamoto, A. Kimura, Y. Miura, M. Shirai, M. Ye, Y. Cui, K. Shimada, H. Namatame, M. Taniguchi, Y. Takeda, Y. Saitoh, E. Ikenaga, S. Ueda, K. Kobayashi, and T. Kanomata, Absence of temperature dependence of the valence-band spectrum of $\mathrm{Co}_{2} \mathrm{MnSi}$, Phys. Rev. B 79, 100405(R) (2009).

[57] S. Ouardi, G. H. Fecher, X. Kozina, G. Stryganyuk, B. Balke, and C. Felser, Symmetry of Valence States of Heusler Compounds Explored by Linear Dichroism in Hard-X-Ray Photoelectron Spectroscopy, Phys. Rev. Lett. 107, 036402 (2011).

[58] K. Sumida, Y. Sakuraba, K. Masuda, T. Kono, M. Kakoki, K. Goto, W. Zhou, K. Miyamoto, Y. Miura, T. Okuda, and A. Kimura, Spin-polarized Weyl cones and giant anomalous Nernst effect in ferromagnetic heusler films, Commun. Mater. 1, 89 (2020).

[59] S. Trudel, G. Wolf, J. Hamrle, B. Hillebrands, P. Klaer, M. Kallmayer, H.J. Elmers, H. Sukegawa, W. Wang, and K. Inomata, Effect of annealing on $\mathrm{Co}_{2} \mathrm{FeAl}_{0.5} \mathrm{Si}_{0.5}$ thin films: A magneto-optical and x-ray absorption study, Phys. Rev. B 83, 104412 (2011).

[60] S. Suga and A. Sekiyama, Photoelectron Spectroscopy. Bulk and Surface Electronic Structures (Springer, Berlin, 2014).

[61] O. Fedchenko, K. Medjanik, S. V. Chernov, D. Kutnyakhov, M. Ellguth, A. Oelsner, B. Schönhense, T. R. F. Peixoto, P. Lutz, C.-H. Min, F. Reinert, S. Däster, Y. Acremann, J. Viefhaus, 
W. Wurth, J. Braun, J. Minár, H. Ebert, H. J. Elmers, and G. Schönhense, 4D texture of circular dichroism in soft-x-ray photoemission from tungsten, New J. Phys. 21, 013017 (2019).

[62] S. V. Chernov, K. Medjanik, C. Tusche, D. Kutnyakhov, S. A. Nepijko, A. Oelsner, J. Braun, J. Minár, S. Borek, H. Ebert, H. J. Elmers, J. Kirschner, and G. Schönhense, Anomalous dlike surface resonances on $\operatorname{Mo}(110)$ analyzed by time-of-flight momentum microscopy, Ultramicroscopy 159, 453 (2015).

[63] G. Schönhense, K. Medjanik, S. Chernov, D. Kutnyakhov, O. Fedchenko, M. Ellguth, D.Vasilyev, A. ZaporozhchenkoZymaková, D. Panzer, A. Oelsner, C. Tusche, B. Schönhense, J. Braun, J. Minár, H. Ebert, J. Viefhaus, W. Wurth, and H. J. Elmers, Spin-filtered time-of-flight k-space microscopy of IR - towards the "complete" photoemission experiment, Ultramicroscopy 183, 19 (2017).

[64] B. Schönhense, K. Medjanik, O. Fedchenko, S. Chernov, M. Ellguth, D. Vasilyev, A. Oelsner, J. Viefhaus, D. Kutnyakhov, W. Wurth, H. J. Elmers, and G. Schönhense, Multidimensional photoemission spectroscopy - the space-charge limit, New J. Phys. 20, 033004 (2018).

[65] D. Kutnyakhov et al., Time- and momentum-resolved photoemission studies using time-of-flight momentum microscopy at a free-electron laser, Rev. Sci. Instrum. 91, 013109 (2020).

[66] M. Kolbe, P. Lushchyk, B. Petereit, H. J. Elmers, G. Schönhense, A. Oelsner, C. Tusche, and J. Kirschner: Highly Efficient Multichannel Spin-Polarization Detection, Phys. Rev. Lett. 107, 207601 (2011).

[67] C. Tusche, M. Ellguth, A. A. Ünal, C.-T. Chiang, A. Winkelmann, A. Krasyuk, M. Hahn, G. Schönhense, and J. Kirschner, Spin resolved photoelectron microscopy using a two-dimensional spin-polarizing electron mirror, Appl. Phys. Lett. 99, 032505 (2011).

[68] D. Kutnyakhov, S. Chernov, K. Medjanik, R. Wallauer, C. Tusche, M. Ellguth, S. A. Nepijko, M. Krivenkov, J. Braun, S. Borek, J. Minár, J. Ebert, H.-J. Elmers, and G. Schönhense, Spin texture of time-reversal symmetry invariant surface states on W(110), Sci. Rep. 6, 29394 (2016).

[69] M. Hahn, G. Schönhense, E. A. Jorge, and M. Jourdan, Significant spin polarization of $\mathrm{Co}_{2} \mathrm{MnGa}$ Heusler thin films on $\mathrm{MgO}(100)$ measured by ultraviolet photoemission spectroscopy, Appl. Phys. Lett. 98, 232503 (2011).

[70] C. Tusche, M. Ellguth, A. Krasyuk, A. Winkelmann, D. Kutnyakhov, P. Lushchyk, K. Medjanik, G. Schönhense, and J. Kirschner, Quantitative spin polarization analysis in photoelectron emission microscopy with an imaging spin filter, Ultramicroscopy 130, 70 (2013).

[71] C. Tusche, A. Krasyuk, and J. Kirschner, Spin-resolved band structure imaging with a high- resolution momentum microscope, Ultramicroscopy 159, 520 (2015).

[72] K. Medjanik, O. Fedchenko, S. N. Chernov, D. Kutnyakhov, M. Ellguth, A. Oelsner, B. Schönhense, T. R. F. Peixoto, P. Lutz, C.-H. Min, F. Reinert, S. Däster, Y. Acremann, J. Viefhaus, W. Wurth, H.-J. Elmers, and G. Schönhense, Direct 3D mapping of the fermi surface and fermi velocity, Nat. Mater. 16, 615 (2017).

[73] K. Medjanik, S. V. Babenkov, S. V. Chernov, D. Vasilyev, B. Schönhense, C. Schlueter, A. Gloskowskii, Y. Matveyev, W. Drube, H. J. Elmers, and G. Schönhense, Progress in HAXPES
Per-formance Combining Full-Field k-Imaging with Time-ofFlight Recording, J. Synchrotron Radiat. 26, 1996 (2019).

[74] S. Babenkov, K. Medjanik, D. Vasilyev, S. V. Chernov, C. Schlueter, A. Gloskovskii, Yu. Matveyev, W. Drube, B. Schönhense, K. Rossnagel, H.-J. Elmers, and G. Schönhense, High-accuracy bulk electronic bandmapping with eliminated diffraction effects using hard X-ray photoelectron momentum microscopy, Commun. Phys. 2, 107 (2019).

[75] See Supplemental Material at http://link.aps.org/supplemental/ 10.1103/PhysRevB.103.054407 for videos showing data arrays of intensity and spin texture and a detailed comparison of experiment and theory for $\mathrm{Co}_{2} \mathrm{MnGa}$.

[76] J. Pierre, R. V. Skolozdra, J. Tobola, S. Kaprzyk, C. Hordequin, M. A. Kouacou, I. Karla, R. Currat, and E. Leliévre-Berna, Properties on request in semi-heusler phases, J. Alloys Compd. 262-263, 101 (1997).

[77] A. Grechnev, I. Di Marco, M. I. Katsnelson, A. I. Lichtenstein, J. Wills, and O. Eriksson, Theory of bulk and surface quasiparticle spectra for Fe, Co, and Ni, Phys. Rev. B 76, 035107 (2007).

[78] P. Klaer, M. Kallmayer, C. G. F. Blum, T. Graf, J. Barth, B. Balke, G. H. Fecher, C. Felser, and H.-J. Elmers, Tailoring the electronic structure of half-metallic heusler alloys, Phys. Rev. B 80, 144405 (2009).

[79] G. H. Fecher, H. C. Kandpal, S. Wurmehl, J. Morais, H.-J. Lin, H.-J. Elmers, G. Schönhense, and C. Felser, Design of magnetic materials: the electronic structure of the ordered, doped heusler compound $\mathrm{Co}_{2} \mathrm{Cr}_{1-x} \mathrm{Fe}_{x} \mathrm{Al}$, J. Phys.: Condens. Matter 17, 7237 (2005).

[80] M. Sargolzaei, M. Richter, K. Koepernik, I. Opahle, H. Eschrig, and I. Chaplygin, Spin and orbital magnetism in full heusler alloys: A density functional theory study of $\mathrm{Co}_{2} Y Z(Y=$ $\mathrm{Mn}, \mathrm{Fe} ; Z=\mathrm{Al}, \mathrm{Si}, \mathrm{Ga}, \mathrm{Ge}$ ), Phys. Rev. B 74, 224410 (2006).

[81] J. Osterwalder, T. Greber, S. Hüfner, and L. Schlapbach, X-Ray Photoelectron Diffraction from a Free-Electron-Metal Valence Band: Evidence for Hole-State Localization, Phys. Rev. Lett. 64, 2683 (1990).

[82] G. S. Herman, T. T. Tran, K. Higashiyama, and C. S. Fadley, Valence Photoelectron Diffraction and Direct Transition Effects, Phys. Rev. Lett. 68, 1204 (1992).

[83] J. Osterwalder, T. Greber, P. Aebi, R. Fasel, and L. Schlapbach, Final-state scattering in angle-resolved ultraviolet photoemission from copper, Phys. Rev. B 53, 10209 (1996).

[84] G. Schönhense and H. C. Siegmann, Transmission of electrons through ferromagnetic material and applications to detection of electron spin polarization, Ann. Phys. 505, 465 (1993).

[85] K. Hild, J. Maul, G. Schönhense, H.-J. Elmers, M. Amft, and P. M. Oppeneer, Magnetic Circular Dichroism in Two-Photon Photoemission, Phys. Rev. Lett. 102, 057207 (2009).

[86] K. Hild, G. Schönhense, H.-J. Elmers, T. Nakagawa and T. Yokoyama, K. Tarafder, and P. Oppeneer, Energy- and angle-dependent threshold photoemission magnetic circular dichroism from an ultrathin $\mathrm{Co} / \mathrm{Pt}(111)$ film, Phys. Rev. B 82, 195430 (2010).

[87] H. C. Kandpal, G. H. Fecher, and C. Felser, Calculated electronic and magnetic properties of the half-metallic, transition metal based Heusler compounds, J. Phys. D 40, 1507 (2007). 